\title{
Noninvasive Vascular Elastography With Plane Strain Incompressibility Assumption Using Ultrafast Coherent Compound Plane Wave Imaging
}

\author{
Jonathan Porée, Damien Garcia, Boris Chayer, Jacques Ohayon, and Guy Cloutier*
}

\begin{abstract}
Plane strain tensor estimation using non-invasive vascular ultrasound elastography (NIVE) can be difficult to achieve using conventional focus beamforming due to limited lateral resolution and frame rate. Recent developments in compound plane wave (CPW) imaging have led to high speed and high resolution imaging. In this study, we present the performance of NIVE using coherent CPW. We show the impact of CPW beamforming on strain estimates compared to conventional focus sequences. To overcome the inherent variability of lateral strains, associated with the low lateral resolution of linear array transducers, we use the plane strain incompressibility to constrain the estimator. Taking advantage of the approximate tenfold increase in frame rate of CPW compared with conventional focus imaging, we introduce a time-ensemble estimation approach to further improve the elastogram quality. By combining CPW imaging with the constrained Lagrangian speckle model estimator, we observe an increase in elastography quality $(\sim 10 \mathrm{~dB}$ both in signal-to-noise and contrast-to-noise ratios) over a wide range of applied strains (0.02 to 3.2\%).
\end{abstract}

Index Terms-Strain imaging, ultrafast coherent plane wave compounding, ultrasound elastography, vascular imaging.

\section{INTRODUCTION}

A CUTE myocardial infarctions and strokes are mostly triggered by the rupture of an atherosclerotic plaque [1]. Unpredictability of the progression of individual plaques, however,

Manuscript received March 27, 2015; revised June 19, 2015; accepted June 22, 2015. Date of publication June 30, 2015; date of current version November 25,2015 . This research was initiated through the support of the Natural Sciences and Engineering Research Council of Canada (NSERC Strategic Grant STPGP-381136-09 and Discovery Grant $138570-11$ ). This program is currently supported by the Collaborative Health Research program of NSERC (\#CHRP 462240-14) and Canadian Institutes of Health Research (\#CPG-134748). Authors acknowledge the scholarship support of MEDITIS-NSERC to Mr. Porée (Institute of Biomedical Engineering, University of Montreal). Asterisk indicates corresponding author.

J. Porée and B. Chayer are with the Laboratory of Biorheology and Medical Ultrasonics, Research Center, University of Montreal Hospital, Montreal, QC, H2X 0A9 Canada.

D. Garcia is with the Laboratory of Biorheology and Medical Ultrasonics, Research Center, University of Montreal Hospital, Montreal, QC, H2X 0A9 Canada, and also with the Research Unit of Biomechanics and Imaging in Cardiology, University of Montreal Hospital, Montreal, QC, H2X 0A9 Canada, and also with the Department of Radiology, Radio-Oncology and Nuclear Medicine, and Institute of Biomedical Engineering, University of Montreal, Montreal, QC, H3C 3J7 Canada.

J. Ohayon is with University of Savoie and Laboratory TIMC-IMAG DyCTiM, CNRS UMR 5525, Joseph Fourier University, 38706 Grenoble, France.

${ }^{*}$ G. Cloutier is with the Laboratory of Biorheology and Medical Ultrasonics, Research Center, University of Montreal Hospital, Montreal, QC, H2X 0A9 Canada, and also with the Department of Radiology, Radio-Oncology and $\mathrm{Nu}$ clear Medicine, and Institute of Biomedical Engineering, University of Montreal, Montreal, QC, H3C 3J7 Canada (e-mail: guy.cloutier@umontreal.ca).

Color versions of one or more of the figures in this paper are available online at http://ieeexplore.ieee.org.

Digital Object Identifier 10.1109/TMI.2015.2450992 makes difficult to anticipate clinical patient outcomes. Non-invasive tools such as computed tomography, magnetic resonance imaging and 3D ultrasound have been developed to evaluate the morphology, tissue content and biological processes involved in the evolution of atherosclerotic plaques [2], [3]. Such methods can quantify plaque volume, stenosis severity, lipid and calcium contents, and the presence of plaque hemorrhage and inflammation [4]. However, although those markers are major risk factors of myocardial infarction and stroke, it is not what causes plaque disruption. Indeed, atherosclerotic plaque rupture is mainly a mechanical process governed by its internal morphology, biological composition and mechanical properties [5]. Pathological and clinical investigations revealed that a plaque with a large thrombogenic lipid core embedded under a thin fibrous cap is more at risk than a dense fibrotic plaque, although the former may present a lower degree of stenosis [6].

Non-invasive vascular elastography (NIVE) has the ability to provide clinicians with mechanical information that could help identify carotid plaques at risk of rupture and thus prevent stroke. Unlike endovascular elastography, NIVE estimates strain maps using an external linear array transducer. Strains in carotid arteries are mostly produced by the intraluminal blood pressure acting perpendicularly to the wall. Tangential motions due to fluid-structure coupling may also intervene [7]. NIVE, however, yields strain maps (i.e., elastograms) into Cartesian coordinates associated with the direction of ultrasound beams. Cross-sectional carotid elastography, which is of interest for 3D reconstruction of a diseased artery, thus ideally requires reliable estimates of strain and shear components, in both axial and lateral directions, that conventional focus imaging cannot provide due to the low lateral resolution of external ultrasound systems. To solve this dilemma, different methods have been proposed to improve strain estimations in the lateral direction [8]-[11]. Konofagou et al. used an iterative scheme involving lateral interpolation of radio-frequency (RF) data [8]. Hansen et al. [10] and another group before them [9] suggested to estimate missing components (i.e., lateral strain and shear) from beam-steered elastography data using different angular compounding schemes.

The relatively low frame rate is another limitation of elastography using standard beamforming. Although conventional systems can provide frame rates around 100 per second, some physiological events, such as the propagation of the pressure wave along the arterial wall at tens $\mathrm{m} / \mathrm{s}$ is likely to impede arterial elastography [12]. More importantly, low frame rates result in large displacements between two consecutive frames and may thus 
increase signal decorrelation [13]. Developments of new beamforming strategies, such as plane wave imaging (PWI) [14], synthetic aperture and sparse array imaging [15], have led ultrasound to provide very high frame rates (ultrafast ultrasound). Synthetic aperture and sparse array imaging were recently proposed to improve NIVE [16]. However, the low transmit power due to single element emission in latter methods might be insufficient for NIVE applications in vivo.

Compound plane wave (CPW) beamforming [14] consists in averaging several PWI-based RF images issued from different tilted emissions to provide high resolution images. CPW can generate a large field of view with similar image quality as conventional beamforming methods [14], [17], but at effective frame rates on the order of hundreds to thousands per second. Such high frame rates can reduce decorrelation artifacts and enable the use of multiple successive frames to perform time-ensemble estimation [18], thus further increasing robustness. In the context of NIVE, Hansen et al. proposed the use of elastograms obtained with beam-steered plane waves and reported results comparable to those of conventional beam-steering techniques [11]. One issue associated with this method is the need of relatively large beam steered angles to perform accurate lateral motion estimations. Large steering angles during plane wave emissions with a linear array transducer, however, may induce significant motion artifacts due to the presence of grating lobes [10].

In the current study, we investigated the effectiveness of CPW beamforming to perform non-invasive vascular elastography using locally affine strain estimators. Since the lateral resolution might still be suboptimal to get reliable strain components, we used the incompressible plane strain assumption as mechanical constraints to increase the precision of the Lagrangian speckle model estimator (LSME) [19]. To further improve its performance and get the full benefit of the ultrafast scheme, we also derived a time-ensemble estimation approach. This new implementation of the LSME was evaluated in simulated and in vitro vessels. In vivo feasibility was also demonstrated in one healthy volunteer. Both conventional (Focus) and CPW data with no mechanical constraints (i.e., without assuming plane strain incompressibility) and no temporal averaging were also gathered for comparison. The goal was to demonstrate that NIVE derived from the mechanically-constrained CPW-based LSME can offer high-quality strain images in the context of carotid plaque analysis.

\section{THEORY}

\section{A. Robust Optical Flow for Strain Estimation Using Plane Strain and Incompressibility Assumptions}

In the field of quasi-static elastography, strain images (elastograms) are usually computed with correlation-based methods [8], [10], [16], phase tracking strategies [20], or least-squaresbased models [19], [21]. The first two sets of methods assume locally uniform displacements and thus do not account for local variations (in axial strain, lateral strain and shear). The main advantage of the LSME least-squares-based model lies in its formulation, which accounts for strain field heterogeneities using local affine transformations. Since it is based on the optical flow constraint requiring intensity constancy through motion [22], this method can be sensitive to speckle decorrelation associated with out-of-plane and high-gradient motions, non-rigid motion of sub-resolution scatterers, and non-uniformity of the ultrasound field [23]. To further complicate matters, in most non-invasive ultrasound systems, the axial resolution is tenfold larger than the lateral one, thus leading to low lateral sensitivity to motion for all abovementioned algorithms.

In the following, we describe a robust implementation of the optical flow method to reduce negative effects of local speckle decorrelation, noise and outlier artifacts. We then introduce a constrained motion model that involves plane strain conditions and tissue incompressibility to overcome the low lateral sensitivity. We finally describe a time-ensemble estimation approach to take advantage of the high frame rate of $\mathrm{CPW}$ imaging to further increase LSME performance.

\section{B. Robust Optical Flow Implementation}

We use, in the following, the notation $(\mathrm{x}, \mathrm{z})$ to describe an image relative to a linear array transducer, where the $x-z$ plane refers to the scan plane: the lateral direction (i.e., $x$-direction) is parallel to the array plane and the axial direction (i.e., z-direction) is perpendicular to it (the elevational y-direction out of the imaging plane is not considered in this study). The fundamental assumption behind optical flow techniques is the brightness constancy of a moving image spot. This leads to the optical flow constraint [24]:

$$
\frac{\partial I}{\partial x} u_{x}+\frac{\partial I}{\partial z} u_{z}+\frac{\partial I}{\partial t}=0
$$

This constraint relates the spatial $(\partial I / \partial x$ and $\partial I / \partial z)$ and temporal $(\partial I / \partial t)$ derivatives of the image intensity to the velocity $\vec{u}=\left(u_{x}, u_{z}\right)^{T}$ at each point of the image. Since there is only one equation for two unknowns, this equation cannot be solved as such. To overcome this problem, small measurement windows (MW) around each pixel were considered, assuming local motion coherence leading to an overdetermined system of $n$ linear equations:

$$
A \vec{u}=b
$$

where $A=\left[\begin{array}{ccc}I_{x_{1}} & \ldots & I_{x_{n}} \\ I_{z_{1}} & \ldots & I_{z_{n}}\end{array}\right]^{T}$ is a $n \times 2$ matrix containing spatial derivatives $\left(I_{x}=\partial I / \partial x\right.$ and $\left.I_{z}=\partial I / \partial z\right)$ and $b=$ $\left[-I_{t_{1}} \cdots-I_{t_{n}}\right]^{T}$ is a $n$ components column vector containing temporal derivatives $\left(I_{t}=\partial I / \partial t\right)$.

The vector flow $\vec{u}$ can be estimated in the least-squares sense using the following:

$$
\vec{u}_{L S}=\left(A^{T} A\right)^{-1} A^{T} b .
$$

In optical flow methods, it is generally better to assign more weight to pixels that are closer to the center of the measurement window. Convenient weights are for instance given by a Gaussian function. The weighted least-squares solution then becomes:

$$
\vec{u}_{W L S}=\left(A^{T} W A\right)^{-1} A^{T} W b
$$


where $W$ is a $n \times n$ diagonal matrix which contains the Gaussian weights. Despite their robustness to white Gaussian noise, leastsquares estimators are usually very sensitive to outliers. In the context of ultrasound elastography, outliers may appear when the intensity conservation through motion is violated or when motion occurs out of the imaging plane. In this case, it is more appropriate to use a least-squares method that is robust against outliers [25].

To minimize their adverse effects, outliers can be assigned a low weight using an iteratively reweighted process. This robust method consists in deriving weights with a specified function by using current residuals and updating them, from iteration to iteration, until residuals remain unchanged. In practice, a few iterative steps ( 3 to 5 ) are sufficient. In the current implementation, at each iteration $k$, residuals $r_{k}$ were computed using the previously estimated vector flow $\vec{u}_{W L S}^{k-1}$ :

$$
r_{k}=A \vec{u}_{W L S}^{k-1}-b
$$

Those residuals were used to recalculate individual weights using, for instance, the bi-square weight function. Components of the linear system ((4)), which led to large residuals, were down-weighted to reduce their influence in the next iteration step (see [22], [25] for more details). After the last iteration, coefficients of determination were computed as:

$$
R_{W L S}^{2}=1-\frac{S S_{r e s}}{S S_{t o t}}
$$

with $S S_{\text {res }}=\sum_{i=1}^{n} w\left(x_{i}\right)\left(A_{i} \vec{u}_{W L S}-b_{i}\right)^{2}$ is the residual sum of squares, $S S_{\text {tot }}=\sum_{i=1}^{n} w\left(x_{i}\right)\left(b_{i}-\bar{b}\right)^{2}$ is the total sum of squares, $\bar{b}$ denotes the mean of $b$ and $w$ is the weight. For each pixel of the estimated motion field, $R_{W L S}^{2}$ gives a goodnessof-fit measure of the motion model $\vec{u}_{W L S}$ on the data.

Besides outliers, optical flow problems are often ill-posed when the support of the estimator (i.e., the size of the MW) is too small and/or when the image intensity is locally too uniform. This may lead to ill-conditioned linear systems where constraint equations do not provide enough information to solve the system accurately. In non-invasive ultrasound elastography, this problem arises mainly due to the low lateral resolution of external probes and the absence of phase information in the lateral direction of images. To overcome this problem, a solution is to use estimators with larger support, at the expense of resolution, or add some constraints to decrease sensitivity to round-off errors. In the following section, we describe the affine motion model used in previous implementations of the LSME [7], [19] and an upgraded motion model assuming incompressible plane-strain elasticity to further constrain the estimation.

\section{Constrained Motion Model}

1) Affine Model: Inside a measurement window, the velocity vector $\vec{u}$ can be approximated by an affine model using its first order Taylor expansion at each pixel point $\overrightarrow{x_{i}}(i=1, \ldots, n)$, which are in the vicinity of the centroid $\overrightarrow{x_{0}}$ of the MW, as:

$$
\vec{u}\left(\overrightarrow{x_{i}}\right)=M\left(\overrightarrow{x_{i}}\right) \cdot \vec{m}\left(\overrightarrow{x_{0}}\right)
$$

Here, $M\left(\overrightarrow{x_{i}}\right)=\left[\begin{array}{llllll}1 & 0 & x & z & 0 & 0 \\ 0 & 1 & 0 & 0 & x & z\end{array}\right]$ and $\vec{m}\left(\overrightarrow{x_{0}}\right)=$ $\left(u_{x}, u_{z}, \Delta_{x x}, \Delta_{x z}, \Delta_{z x}, \Delta_{z z}\right)^{T}$ is the motion model vector, where $\Delta_{i j}=\partial u_{i} / \partial_{j}$. Vector components $\Delta_{x x}$ and $\Delta_{z z}$ represent the lateral and axial strains (spatial domain), and $\Delta_{x z}$ and $\Delta_{z x}$ stand for the lateral and axial shear, respectively. The robust least-squares estimator in (4) thus becomes:

$$
\begin{gathered}
\vec{m}_{W L S}=\left(A^{T} W A\right)^{-1} A^{T} W b \\
\text { with } A=\left[\begin{array}{cccccc}
I_{x_{1}} & I_{z_{1}} & x_{1} I_{x_{1}} & z_{1} I_{x_{1}} & x_{1} I_{z_{1}} & z_{1} I_{z_{1}} \\
\vdots & & & \vdots & \\
I_{x_{n}} & I_{z_{n}} & x_{n} I_{x_{n}} & z_{n} I_{x_{n}} & x_{n} I_{z_{n}} & z_{n} I_{z_{n}}
\end{array}\right]
\end{gathered}
$$

This estimator returns both displacements $\vec{u}=\left(u_{x}, u_{z}\right)^{T}$ and the $2 \mathrm{D}$ velocity gradient matrix $\Delta=\left[\Delta_{i j}\right]$ at each point of the image plane. Assuming small deformations between two consecutive ultrasound images, one can derive the strain tensor $\varepsilon$ from the matrix $\Delta$ using the following relation:

$$
\varepsilon=\frac{1}{2}\left(\Delta+\Delta^{T}\right) \Delta t
$$

where $\Delta t$ is the time delay between two consecutive frames.

2) Plane Strain Incompressibility Model: Ex vivo analyses have confirmed that arteries may be considered incompressible under physiologic conditions [26]. Healthy arteries are also known to behave as thick-walled circular cylinders in a state of plane strain [27], where cross-sectional strains preponderate. This condition is often taken for granted in vascular biomechanics [28]-[31]. It is unknown, however, at which stage of the continuum of atherosclerotic disease the plane-strain state might be violated. In vascular elastography, the transverse plane-strain condition can be conveniently assumed in most situations, as long as 1) longitudinal dimensions of wall tissues are greater than their transverse dimensions [32], 2) the internal blood pressure acts mostly in the transverse (imaging) plane, and 3) longitudinal motions due to fluid-structure interactions can be neglected. Under these conditions, we can assume that non-rigid out-of-plane motions are negligible on the transverse imaging plane. In this regard, small out-of-plane deformations should little affect the speckle patterns since the elevational resolution, defined by the elevational F-number $=z_{f} / b_{h}=16 / 4$, where $z_{f}$ is the focal depth and $b_{h}$ the element height (see Eq. 3.30 in [33]), is about 4 wavelengths for a linear array transducer.

In a transverse plane-strain state, strains occurring in the direction of the vessel axis $\vec{y}$ (i.e., $\varepsilon_{y y}, \varepsilon_{x y}, \varepsilon_{z y}$ ) can be neglected. The full 3D strain tensor thus reduces to:

$$
\boldsymbol{\varepsilon}=\left(\begin{array}{lll}
\varepsilon_{x x} & \varepsilon_{x y} & \varepsilon_{x z} \\
\varepsilon_{x y} & \varepsilon_{y y} & \varepsilon_{z y} \\
\varepsilon_{x z} & \varepsilon_{z y} & \varepsilon_{z z}
\end{array}\right) \approx\left(\begin{array}{ccc}
\varepsilon_{x x} & 0 & \varepsilon_{x z} \\
0 & 0 & 0 \\
\varepsilon_{x z} & 0 & \varepsilon_{z z}
\end{array}\right)
$$

Incompressibility also states that tissues subject to stresses do not vary in volume. In our context, it results in:

$\operatorname{div}(\vec{u} \Delta t)=\left(\frac{\partial u_{x}}{\partial x}+\frac{\partial u_{y}}{\partial y}+\frac{\partial u_{z}}{\partial z}\right) \Delta t=\varepsilon_{x x}+\varepsilon_{y y}+\varepsilon_{z z} \approx 0$ 
From (10), it thus follows that:

$$
\varepsilon_{x x} \approx-\varepsilon_{z z}
$$

The affine transformation ((8)) modelling the non-rigid motion finally reduces to:

$$
\vec{u}_{i n c}\left(\overrightarrow{x_{i}}\right)=M_{i n c}\left(\overrightarrow{x_{i}}\right) \cdot \vec{m}_{i n c}\left(\overrightarrow{x_{0}}\right)
$$

where $M_{\text {inc }}\left(\overrightarrow{x_{i}}\right)=\left[\begin{array}{ccccc}1 & 0 & z & 0 & -x \\ 0 & 1 & 0 & x & z\end{array}\right]$ and $\vec{m}_{i n c}\left(\overrightarrow{x_{0}}\right)=$ $\left(u_{x}, u_{z}, \Delta_{x z}, \Delta_{z x}, \Delta_{z z}\right)^{T}$. In the current implementation, the lateral strain $\varepsilon_{x x}$ was computed from the axial strain $\varepsilon_{z z}$ using (12).

\section{Time-Ensemble Estimation}

One of the main benefits of CPW beamforming when compared with conventional focused imaging is the increase in frame rate by tenfold or higher. High frame rates reduce the time step $\Delta t$ between two consecutive frames. Even if this may be seen as an additional challenge for most motion estimators, reducing $\Delta t$ decreases the occurrence of speckle decorrelation and intensity variation through motion. In addition, an increased frame rate allows analysis of frame ensembles to reduce the variance of motion estimates, as it has been proposed in particle image velocimetry [34], or the use of the temporal information in the motion model framework to improve robustness, as proposed here.

Using the optical flow method described in the previous section, time-ensemble estimation can be done by combining successive pairs of frames to increase the number of optical flow equations ((1)). By doing so, we assume that the motion field $\vec{u}\left(\overrightarrow{x_{i}}, t_{i} ; \overrightarrow{x_{0}}, t_{0}\right)$, centered on $\left(\overrightarrow{x_{0}}, t_{0}\right)$, is fully described by (14) over a given $\mathrm{MW}_{\mathrm{t}}$ (i.e., an $\mathrm{MW}$ within the ROI of successive frames):

$$
\vec{u}\left(\overrightarrow{x_{i}}, t_{i} ; \overrightarrow{x_{0}}, t_{0}\right)=M\left(\overrightarrow{x_{i}}\right) \cdot \vec{m}\left(\overrightarrow{x_{0}}\right)
$$

In this model, the motion $\vec{m}$ is assumed to be constant over a given period $T=n_{t} . \Delta t$, where $n_{t}$ is the number of successive frame pairs. This leads to an overdetermined system of $n_{t} \times n$ linear equations, where $n$ is the number of pixels over the spatial support of the estimator (MW). Extending the support of the estimator over time thus increases the number of constrained optical flow equations ((1)) by a factor $n_{t}$, which improves its robustness to noise. Formulated as a least squares estimator, the covariance of the estimator reads as follows (see (5) in [35]):

$$
C O V=\left(A^{T} W A\right)^{-1} \frac{\sigma^{2}}{n_{t} \times n-p}
$$

where $\sigma^{2}$ is the variance of the noise and $p$ the number of variables describing the model (i.e., $p=6$ for the affine model and $p=5$ for the plane strain incompressibility model). Note that if one would consider averaging $n_{t}$ successive and independent estimates, the covariance reads as $C O V$ $=\left(A^{T} W A\right)^{-1} \sigma^{2} /\left(n_{t} n-n_{t} p\right)$, which would be less efficient.

\section{E. Lagrangian Speckle Model Estimator Implementation}

The LSME algorithm was implemented in Matlab 7.10 (MathWorks Inc., Natick, MA, USA). As summarized in Fig. 1, it contains 7 steps:

Step 1) Manual segmentation of the vascular wall on the first frame to generate a region of interest (ROI) and propagation of the ROI on remaining frames using speckle tracking. This step can be automatized for cross-sectional ultrasound images using the robust algorithm described in [36].

Step 2) Subdivision of RF images within the ROI into small measurement windows (MW), defined by their size and overlap.

Step 3) Estimation of subpixel rigid motion $\vec{U}$ for each MW, using Fourier-based ensemble-correlations [34] to account for large displacements. The corresponding correlation coefficients $\left(R_{\text {corr }}^{2}\right)$ are stored.

Step 4) Robust low-pass filtering (i.e., smoothing+additional outlier removal) of the rigid motion field using correlation coefficients $\left(R_{\text {corr }}^{2}\right)$ as regularization weights (see [37] for more details).

Step 5) Pixelwise rigid registration between pre- and postmotion MWs using the displacement field $\vec{U}$ determined in step 4.

Step 6) For each MW (or $\mathrm{MW}_{\mathrm{t}}$ ), computation of the affine motion vector $\vec{m}$ from registered pre- and post-motion MWs given in step 5, using the robust leastsquares method (see (4)).

Step 7) Robust smoothing of strain tensor components yielded in step 6 , using the product of the correlation coefficients $\left(R_{\text {corr }}^{2}\right.$, see step 3$)$ and the coefficients of determination $\left(R_{W L S}^{2}\right.$, see (6)) as regularization weights.

The spatial support of the optical flow estimator was a $2 \mathrm{D}$ Gaussian function with full widths at half maximum (FWHM) set at $1 \mathrm{~mm}$ and size set at $1.7 \mathrm{~mm}$, for both axial and lateral directions. A window overlap of $80 \%$ was used. To evaluate the performance of the time-ensemble estimation (see Section IV-C-2), we used $n_{t}=8$ successive frame pairs, otherwise we used $n_{t}=1$.

\section{Materials AND Methods}

We now describe the methodology adopted to evaluate the performance of the new estimator on both conventional focus (Focus) and compound plane wave (CPW) data. The constrained estimator (plane strain+incompressibility, see (13)) was evaluated in silico, in vitro and in vivo, and compared with the unconstrained estimator $((7))$. The results associated with the unconstrained estimators are referred to as Focus and $\mathrm{CPW}$, whereas those corresponding to constrained methods are labeled Focus+Inc and CPW+Inc.

\section{A. Simulations}

A finite element method (FEM) coupled with ultrasound simulations were used to mimic geometries of carotid arteries as acquired in a cross-sectional view. The FEM was used to simulate wall motion under physiological pressure conditions and ultrasound simulations aimed at mimicking RF speckle patterns. 


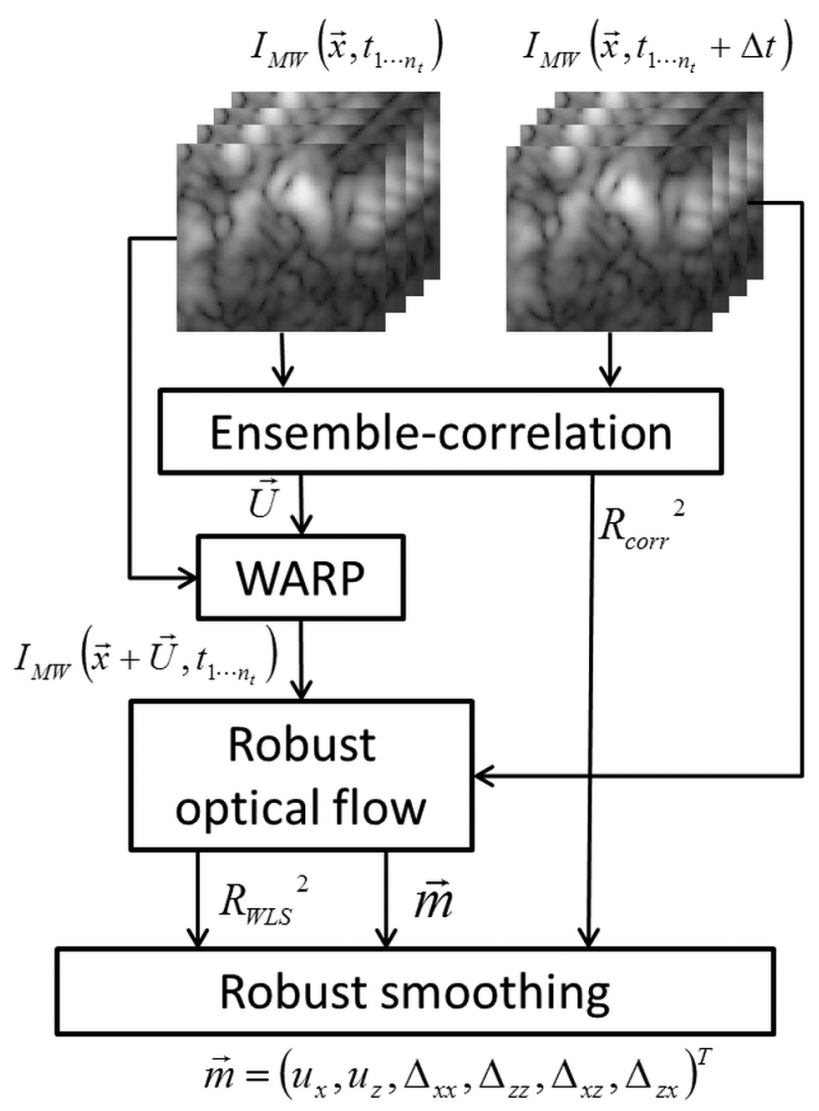

Fig. 1. Block diagram of the algorithmic implementation assessing the plane strain tensor. A coarse pixelwise rigid registration using 2D Fourier-based ensemble-correlation (see [34]) is first performed between pre- and post-deformed RF images, $I_{M W}\left(\vec{x}, t_{1 \cdots n_{t}}\right)$ and $I_{M W}\left(\vec{x}, t_{l \cdots n_{t}}+\Delta t\right)$, to get the registered pre-deformed images $I_{M W}\left(\vec{x}+\vec{U}, t_{1 \cdots n_{t}}\right)$. The unknown motion model vector $\vec{m}$ is then computed from registered pre-deformed and post-deformed images, using a robust optical flow method. $R_{c o r r}^{2}$ and $R_{W L S}^{2}$ are, respectively, the ensemble-correlation values computed from the rigid registration step, and the coefficient of determination evaluated using the robust optical flow. The product of those quality measures are finally used as weighting coefficients for the robust smoothing procedure, which is performed using the algorithm described in [37].

RF echo frames were used to compute elastograms using the four abovementioned approaches (Focus, CPW, Focus + Inc and $\mathrm{CPW}+\mathrm{Inc})$. The following subsections describe the simulation process.

1) Finite Element Modeling of Vessel Phantoms: Models of one healthy and one pathological carotid arteries were created with COMSOL Multiphysics (Structural Mechanics Module, version 3.5, COMSOL, France). The healthy model had inner and outer radii of $1.5 \mathrm{~mm}$ and $6 \mathrm{~mm}$, respectively. The vessel wall had a uniform Young modulus $\mathbf{E}$ of $300 \mathrm{kPa}$, which is close to the elasticity of non-fibrotic tissue under physiological constraints [38]. The plaque model described in [11] had a large soft necrotic core $(\mathbf{E}=20 \mathrm{kPa})$ embedded in a homogeneous medium $(\mathbf{E}=1000 \mathrm{kPa})$ and covered by a thin fibrous cap $(\mathbf{E}=1500 \mathrm{kPa})$. Each medium was considered isotropic and quasi-incompressible (Poisson's ratio $\gamma=0.499$ ). To prevent rigid body translations, a soft $(\mathbf{E}=1 \mathrm{~Pa})$ and compressible $(\gamma=0.001)$ layer was added on the outer layer of the two carotid models. The outer contour of this artificial layer was anchored and was not considered in the strain analysis. Displacement fields were calculated for an intraluminal pressure increase of $0.5 \mathrm{kPa}(\sim 4 \mathrm{mmHg})$ between two successive RF frames under plane strain conditions. The reference strain fields were computed from axial and lateral displacements yielded by FEM simulations.

2) Acoustic Models: Ultrasound backscatter models were created by randomly distributing point scatterers over the crosssection of the vessel wall. To ensure fully developed speckle, the scatterer density was chosen as 100 per resolution cell [39]. Displacement fields generated by COMSOL were applied on scatterers to create post-deformation acoustic models of arteries. The Field II simulation program [40] was used to mimic the 128 elements of a L14-5/38 linear array probe (Ultrasonix Medical Corporation, Richmond, BC, Canada) with a 7.2 MHz center frequency, $70 \%$ fractional bandwidth $($ at $-6 \mathrm{~dB})$ and a sampling rate of $40 \mathrm{MHz}$. The transducer produced either focused beams, using a 64-element aperture in transmission and a focus at 16 $\mathrm{mm}$ (corresponding to the center of the vessel) for conventional imaging, or steered plane waves for CPW imaging. For the CPW sequence, steering angles $\alpha$ were varied from $-\alpha_{\max }$ to $\alpha_{\max }$ with an angular step $\Delta \alpha$ between each steer. All 128 elements of the aperture were active during plane wave transmission. CPW data were beamformed off-line using the delay-and-sum algorithm [14]. For each set of simulated data, white Gaussian noise was added to produce ultrasound images with a realistic signal-to-noise ratio (SNR) of $20 \mathrm{~dB}$, as in [16]. The SNR measured from in vivo data described later was $\sim 25 \mathrm{~dB}$.

\section{B. Phantom Study}

1) Phantom Fabrication: In vitro validation was performed on polyvinyl alcohol cryogel tissue mimicking vessel phantoms. These phantoms were constructed as described in [41]. The first phantom was built to model a homogenous vessel wall as in the simulation study. It was set in a mold that was $150 \mathrm{~mm}$ long and had inner and outer radii of $1.5 \mathrm{~mm}$ and $6 \mathrm{~mm}$, respectively. It underwent 6 freeze-thaw cycles (from $-20^{\circ} \mathrm{C}$ to $20^{\circ} \mathrm{C}$ ) of 24 hours each.

The second phantom was built to mimic a plaque similar to the simulation geometry (see Fig. 2). The inner layer was 0.5 $\mathrm{mm}$ thick and underwent 8 freeze-thaw cycles to mimic a hard fibrous cap. The outer layer underwent 6 freeze-thaw cycles to mimic the media-adventitia, and the inclusion underwent 1 freeze-thaw cycle to represent a soft lipid pool. Such fabrication processes were reported to provide stiffnesses of $465 \pm 53$ $\mathrm{kPa}$ ( 8 freeze-thaw cycles), $322 \pm 37 \mathrm{kPa}$ (6 cycles) and $25 \pm$ $3 \mathrm{kPa}$ (1 cycle) [41].

2) Test Bench: Vessel phantoms were suspended in a bath and pressurized with a water column filling the lumen (see Fig. 2). To evaluate the impact of the beamforming strategy (i.e., CPW vs. conventional focusing), without considering the difference in frame rate, a static analysis was performed. The intra-luminal pressure was decreased incrementally from 120 $\mathrm{mmHg}$ down to $80 \mathrm{mmHg}$ using $4 \mathrm{mmHg}$ steps by lowering the water column height. Conventional focus and compound plane wave data were acquired at each pressure step, as described in the following Section III-B-3. 


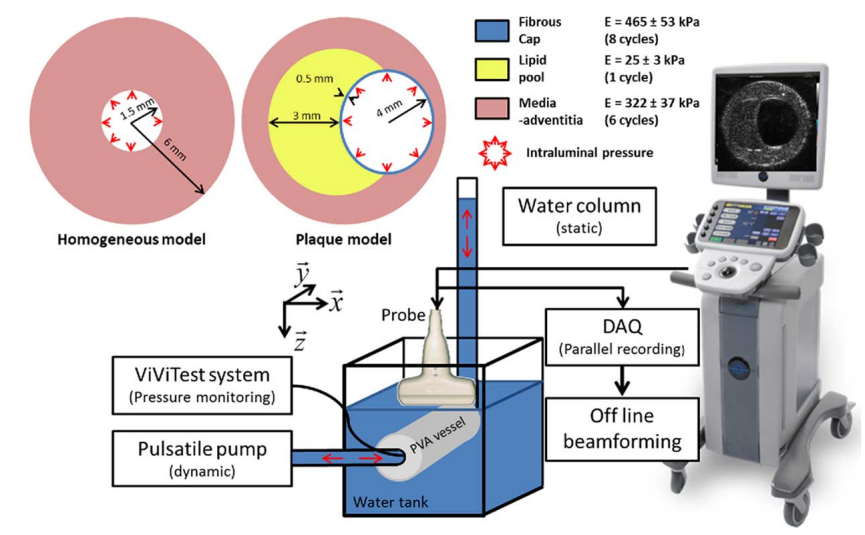

Fig. 2. Schematic illustration of the experimental setup used for the in vitro phantom validation. The water column was used to incrementally increase the intraluminal pressure, with successive pressure steps of $4 \mathrm{mmHg}(\sim 0.5 \mathrm{kPa})$. Alternatively, the pulsatile pump was used to increase dynamically the intraluminal pressure from $60 \mathrm{mmHg}$ to $120 \mathrm{mmHg}$ and evaluate the impact of the frame rate.

To evaluate the impact of the time-ensemble estimation approach (see Section II-D), an unsteady analysis was also conducted on the homogeneous vessel phantom. The intraluminal pressure was dynamically increased, from $60 \mathrm{mmHg}$ to 120 mmHg, using a pulsatile pump (model 1421, Harvard Aparatus, Holliston, MA, USA) and monitored using a ViVitest software system (Vivitro Labs Inc., Victoria, BC, Canada). To demonstrate the robustness of the estimator over a wide range of applied strains (Fig. 8), the time step between frames was varied from $\Delta t$ to $128 \Delta t$ leading to a wide range of applied pressures (the pressure increment $\Delta P$ between pairs of frames varied proportionally with $m \Delta t, m=1$ to 128 ). For both static and dynamic tests, specular reflections at the interface of vessel phantoms were reduced by using degassed saline ( $5 \%$ salt) within the water column and container to match acoustic impedances, as described in [10].

3) Data Acquisition: RF data were acquired using a SonixTouch ultrasonic system (Ultrasonix Medical Corp., Richmond, $\mathrm{BC}$, Canada) equipped with a 128-element linear array probe (L14-5/38) and the SonixDAQ multi-channel system. The scanner was programmed using a software development kit (TexoSDK, v6.0.1) to generate and record plane wave data. Focused and plane wave data were recorded successively using the same parameters as in the simulations. RF data were beamformed (see Section III-D below) and resulting RF images were processed with the proposed estimator to get elastograms.

\section{In Vivo Feasibility}

To evaluate the four motion estimators in vivo, the carotid artery of a 30-year-old male without known cardiovascular disease history was imaged. Both conventional focus (Focus) and compound plane wave (CPW) RF sequences were successively recorded as in the in vitro study, using the SonixTouch scanner. The protocol was approved by the human ethical review committee of the University of Montreal Hospital Research Center, and the volunteer signed an informed consent.

\section{Image Reconstruction (in vitro and in vivo)}

As performed with simulations, coherent plane wave compounding images were recovered by applying the delay-and-sum technique and steering angles $\alpha$ varying from $-\alpha_{\max }$ to $\alpha_{\max }$ with an angular step $\Delta \alpha$. In reception, the aperture was dynamically adapted using an F-number set to 1.75 , as in [14]. All RF echo frames were reconstructed on a regular grid that had a lateral sampling of 20 columns $/ \mathrm{mm}$ (total $=768$ columns) and an axial sampling frequency of 52 rows $/ \mathrm{mm}$ (total $=1580$ rows). No specific image reconstruction was necessary for standard beamforming (Focus) since we used the pre-programmed scheme of the SonixTouch scanner.

\section{E. Data Analysis}

1) Cartesian to Principal Strain Transformation: Cartesian strain tensors were transformed into local principal strain tensors $\boldsymbol{\varepsilon}_{p}=\left(\begin{array}{cc}\varepsilon_{\max } & 0 \\ 0 & \varepsilon_{\min }\end{array}\right)$, using the following equation (see [42], p. 100):

$$
\varepsilon_{\text {max }, \min }=\frac{\varepsilon_{x x}+\varepsilon_{z z}}{2} \pm \sqrt{\left(\frac{\varepsilon_{x x}-\varepsilon_{z z}}{2}\right)^{2}+\left(\varepsilon_{x z}\right)^{2}}
$$

The principal strain orientation was deduced from the following expression:

$$
\theta_{p}=\frac{1}{2} \tan ^{-1}\left(\frac{2 \varepsilon_{x z}}{\varepsilon_{x x}-\varepsilon_{z z}}\right)
$$

Note that for the specific case of a circular homogeneous vessel wall subjected to an internal pressure increase, the maximum and minimum principal strains are equal to the circumferential and radial strains, respectively.

2) RF Data Quality Measurements: To investigate how beamforming (CPW vs. conventional focus) can affect RF images used to compute elastograms, we evaluated the SNR and signal-to-interference ratio (SIR) of reconstructed B-mode images using:

$$
\begin{aligned}
S N R & =10 \log \left(\frac{P_{\text {signal }}}{P_{\text {noise }}}\right) \\
S I R & =10 \log \left(\frac{P_{\text {signal }}}{P_{\text {interferences }}}\right)
\end{aligned}
$$

where $P_{\text {signal }}$ is the average intensity over the vessel wall, and $P_{\text {noise }}$ and $P_{\text {inter ferences }}$ are the average noise and grating lobe interference intensities over regions described in Fig. 3. This evaluation was performed on the homogeneous vessel phantom mimicking a normal carotid artery.

3) Elastogram Quality Measurements: The quality of estimated strain maps was also evaluated on the homogeneous vessel phantom using two metrics: 1) the elastographic signal-to-noise ratio $\left(\mathrm{SNR}_{\mathrm{e}}\right)$, and 2) the contrast-to-noise ratio $\left(\mathrm{CNR}_{\mathrm{e}}\right)$. Due to the circular symmetry of the homogeneous phantom, principal strains $\varepsilon_{\max }$ and $\varepsilon_{\min }$ are of opposite signs and follow an inverse-square law relation with distance (i.e., strains are inversely proportional to $r^{2}$ ), as already described [10]. Thus, for a given radial distance $r$, strains are expected to be constant over the circumferential direction. It was thus 

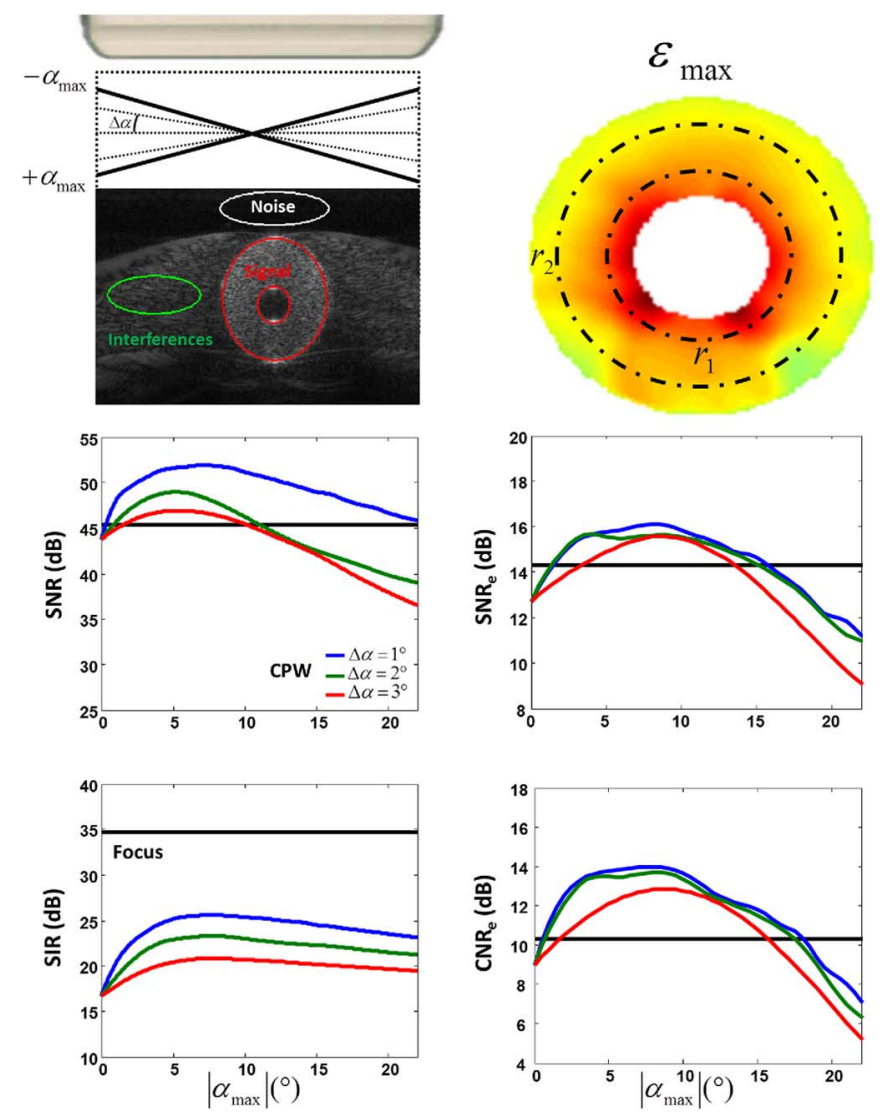

Fig. 3. (Top left) Reconstructed B-mode image of the mimicked normal artery, identified with red circles, surrounded by water. We intentionally presented here a worst case scenario (i.e., no compounding corresponding to $\left|\alpha_{\max }\right|=$ $\left.0^{\circ}\right)$ to emphasize speckle outside the vessel attributed to grating lobe interferences. (Top right) Principal maximum strains and radii used in the definition of Eqs. (20) and (21). (Left) Signal-to-noise ratio (SNR) and signal-to-interference ratio (SIR) of beamformed in vitro B-mode images. (Right) Elastographic signal-to-noise ratio $\left(\mathrm{SNR}_{\mathrm{e}}\right)$ and contrast-to-noise ratio $\left(\mathrm{CNR}_{\mathrm{e}}\right)$ as a function of the absolute value of the maximum steering angle $\alpha_{\max }$ and angular step $\Delta \alpha$. Straight black lines on each panel display results with the standard focus method.

possible to calculate the $\mathrm{SNR}_{\mathrm{e}}$ and $\mathrm{CNR}_{\mathrm{e}}$, in decibels, using points located on circles around the lumen center axis using:

$$
\begin{aligned}
& S N R_{e}=10 \log \left(\frac{\mu_{r_{1}}}{\sigma_{r_{1}}}\right)+10 \log \left(\frac{\mu_{r_{2}}}{\sigma_{r_{2}}}\right), \text { and } \\
& C N R_{e}=10 \log \left(\frac{2\left(\mu_{r_{1}}-\mu_{r_{2}}\right)^{2}}{\sigma_{r_{1}}^{2}+\sigma_{r_{2}}^{2}}\right)
\end{aligned}
$$

Here, $\mu_{r_{1}}, \mu_{r_{2}}$ and $\sigma_{\mathrm{r}_{1}}, \sigma_{\mathrm{r}_{2}}$ are means and standard deviations computed along circles of radii $r_{1}=2.5 \mathrm{~mm}$ and $r_{2}=4 \mathrm{~mm}$ with respect to the lumen center axis (see Fig. 3). Those radii were selected at $1 \mathrm{~mm}$ of inner and outer vessel wall contours to avoid truncated MWs. Means and standard deviations on each circle were estimated using 2D linear interpolation of the maximum principal strain $\varepsilon_{\max }$.

\section{RESUlTS}

\section{A. Impact of $C P W$ Beamforming Parameters on in vitro B-Mode and Strain Map Image Quality}

Using plane wave imaging, averaging the contribution of several steered plane waves through compounding was shown to improve the B-mode image contrast and lateral resolution [14], [17], [43]. Our data set (Fig. 3) shows that varying the steering angle between $\pm 22^{\circ}$ with angular steps $\Delta \alpha$ of 1,2 or $3^{\circ}$ had a strong impact on noise levels (SNR) and grating lobe interferences (SIR) of B-mode images. Those parameters also influenced $\mathrm{SNR}_{\mathrm{e}}$ and $\mathrm{CNR}_{\mathrm{e}}$ of strain maps. According to Fig. 3, optimum parameters to improve the quality of elastograms were $\left|\alpha_{\max }\right|=10^{\circ}$ and $\Delta \alpha=1^{\circ}$ (corresponding to 21 plane wave transmissions). This combination outperformed focus imaging despite its lower performance in term of grating lobe interference (see SIR in Fig. 3). This combination of parameters was used to evaluate CPW imaging in the rest of this study (i.e., in silico, in vitro and in vivo results).

\section{B. Performance Assessment of Strain Maps With Simulations}

Elastograms computed on simulated RF data with the proposed constrained estimator provided good match with FEM ground-truth solutions (Fig. 4), for both beamforming techniques (Focus+Inc and $\mathrm{CPW}+\mathrm{Inc}$ ). The constrained estimator performed particularly well at 3 and 9 o'clock for $\varepsilon_{\text {min }}$, and at 6 and 12 o'clock for $\varepsilon_{\text {max }}$. In those regions, principal strains are mostly aligned with the lateral strain component $\varepsilon_{x x}$, as can be seen from the strain orientation $\theta_{p}$ in Fig. 4. Without plane strain incompressibility, those strains are more sensitive to the low lateral resolution. Note that for circular geometries subject to a positive stress (i.e., $\Delta P>0$ ), maximum and minimum principal strains correspond to circumferential and radial strains, respectively. For the plaque geometry, all maximum principal strain maps revealed the presence of the soft lipid pool. The contour of the soft inclusion, however, was better delineated on $\varepsilon_{\min }$ with the constrained estimator (Focus+Inc and $\mathrm{CPW}+\mathrm{Inc})$. Visually, CPW outperformed conventional focus imaging, with or without mechanical a priori assumptions. This is better appreciated in Fig. 5 where percentiles of principal strains are plotted for each method as a function of radial distances (homogeneous phantom). Conventional focus beamforming gave largest variances, while CPW with mechanical constraints provided best results. As expected from the theory of a thick-walled cylinder subjected to an internal pressure (as described in [10]), principal strains in the homogeneous phantom varied inversely with the squared radial distance, (Fig. 5). Regarding the plaque geometry, estimated strain profiles (Fig. 6) along plaque shoulders (A \& C) and fibrous cap (B) revealed good accuracy with ground truth. Again, the constrained strain estimator associated with CPW imaging (CPW+Inc) gave the best results.

\section{Performance Assessment of Strain Maps With In Vitro Data}

1) Static Analysis: Elastograms obtained from in vitro data confirmed observations with simulations. The Focus method provided inconsistent principal strains at 3 and 9 o'clock for $\varepsilon_{\min }$, and 6 and 12 o'clock for $\varepsilon_{\max }$, very likely due to the limited lateral resolution (Fig. 7). Adding mechanical constraints improved considerably strain estimates, giving results more consistent with theory (as displayed in Fig. 4). Regarding the plaque geometry, the contour of the soft inclusion was easier to delineate when using the constrained strain estimator. In concordance with the simulation study, CPW imaging performed 


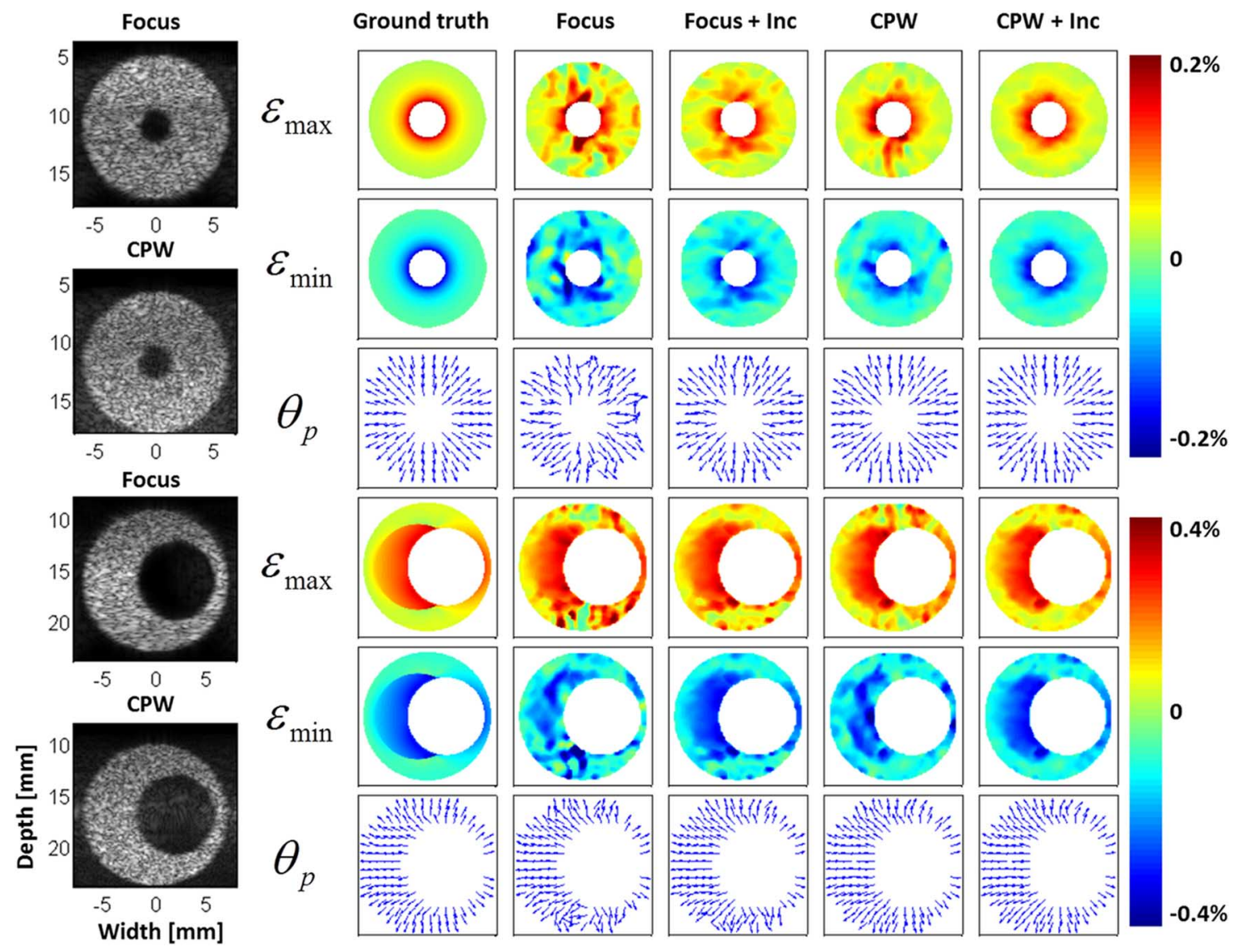

Fig. 4. B-mode images (first column), maximum, minimum and orientation of the principal strain tensor $\left(\varepsilon_{\max }, \varepsilon_{\min }, \theta_{p}\right)$ computed with the finite element model (ground truth), and with four beamforming strategies (Focus, Focus+Inc, CPW, CPW+Inc). The first three rows display results of the simulated concentric homogeneous vessel, whereas the last three rows correspond to results of the plaque geometry.

better than conventional focus imaging, and the addition of the constrained estimator provided best results.

2) Dynamic Analysis: The $\mathrm{SNR}_{\mathrm{e}}$ and $\mathrm{CNR}_{\mathrm{e}}$ analysis (Fig. 8), over a wide range of applied strains (proportional to the internal lumen pressure $\Delta P$ ), confirmed the qualitative observations of Fig. 7. Overall, CPW imaging performed better than conventional focusing. However, regardless of the beamforming method (i.e., either Focus or CPW imaging), strain quality (assessed with $\mathrm{SNR}_{\mathrm{e}}$ and $\mathrm{CNR}_{\mathrm{e}}$ ) generally decreased, when strains were estimated using a single pair of images (i.e., no time-ensemble estimation), for applied strains lower than $0.2 \%$, approximately. With the proposed time-ensemble estimation available in ultrafast mode (i.e., CPW), high-quality elastograms over a wide range of applied strains were obtained using $n_{t}=8$ ensemble pairs (Fig. 8). The time-ensemble $\mathrm{CPW}+$ Inc method provided highest $\mathrm{SNR}_{\mathrm{e}}$ and $\mathrm{CNR}_{\mathrm{e}}$ values over the whole range of applied strains (displayed between $10^{-2}$ to $10^{1} \%$ ). Note that because the frame rate of focus imaging differed from that of the $\mathrm{CPW}$ sequence, the range of applied strains on the $x$-axis (Fig. 8) is slightly different $(0.04 \%$ to $2.8 \%$ for Focus and $0.02 \%$ to $3.2 \%$ for $\mathrm{CPW}$ ).

\section{In Vivo Feasibility in One Healthy Volunteer}

In vivo elastograms obtained in the healthy volunteer were consistent with simulation and in vitro findings (Fig. 9). Minimum and maximum cumulated principal strains displayed negative and positive deformations at the peak of the cumulated strain curve (frame \#10, Fig. 10) (i.e., compression and dilatation), respectively, as expected for this phase of the cardiac cycle. The local orientation $\theta_{p}$ of the strain tensor, however, was not always consistent (i.e. not always oriented radially) for unconstrained estimators.

In contrast, constrained estimators (Focus+Inc and $\mathrm{CPW}+\mathrm{Inc}$ ) provided more consistent and reproducible results (over the two trials), with strain tensors mostly oriented in the radial direction. The reproducibility of the CPW method can be better appreciated in Fig. 10 where the spatially averaged cumulated maximum principal strain followed the same trend over the two trials, except at peak systole where they differed by only $\sim 1 \%$. Those curves look like systemic blood pressure curves, as can be measured using photoplethysmography [44], which was expected for a normal artery. In accordance with in silico and in vitro results, CPW imaging provided more reproducible results then standard focusing, with or without the plane strain incompressibility assumption.

\section{DISCUSSION}

In this study, the Lagrangian Speckle Model Estimator (LSME), previously developed with proven clinical feasibility in the context of longitudinal image acquisitions [7], [21], [45], was adapted to compute the $2 \mathrm{D}$ plane strain tensor of cross-sectional artery views. To overcome the limited lateral resolution of linear array transducers in the context of NIVE, we proposed a constrained version of the optical flow using the plane-strain 

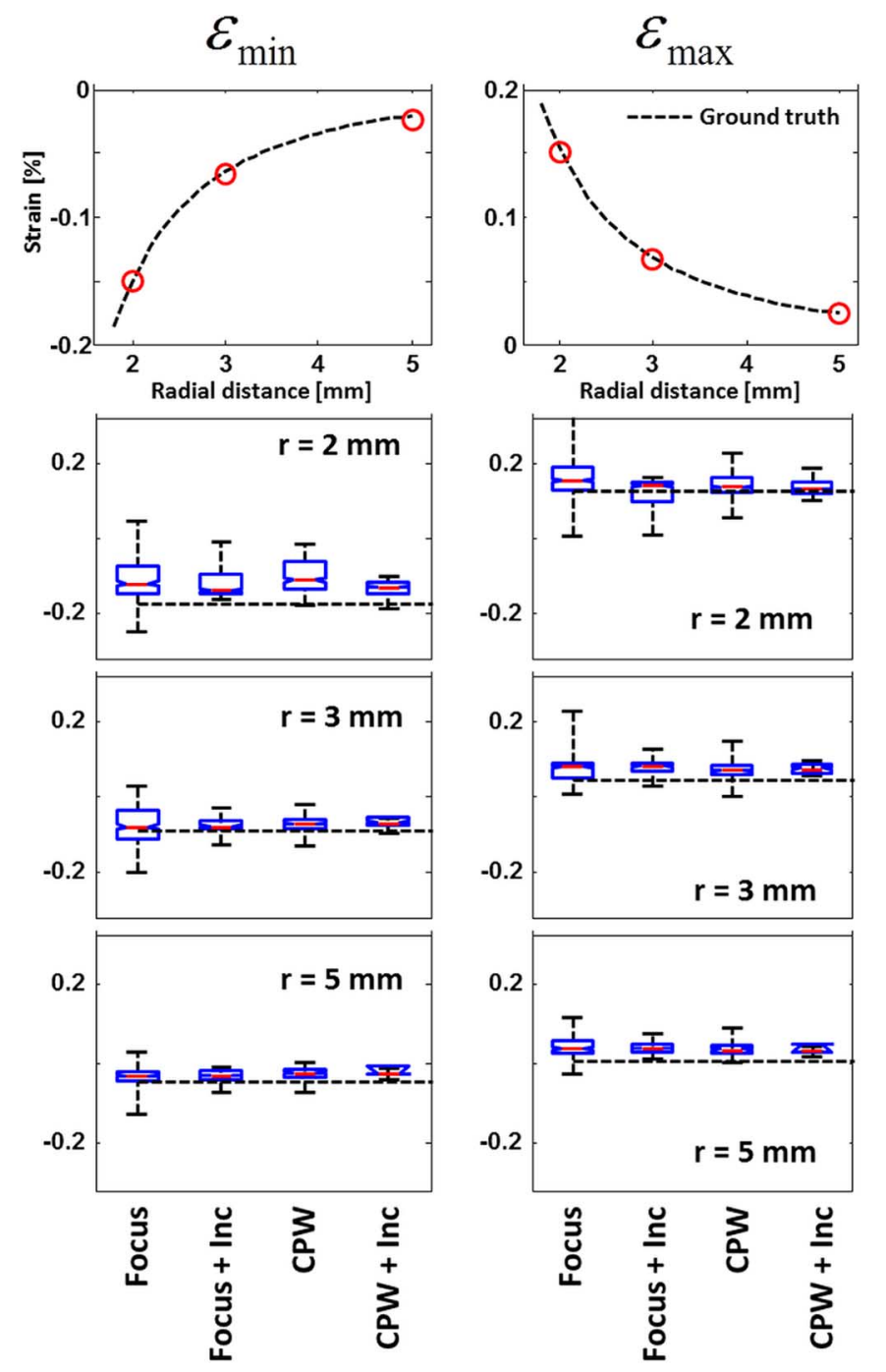

Fig. 5. Boxplots (median, 25th and 75th percentiles) of minimum and maximum principal strains $\left(\varepsilon_{\min }, \varepsilon_{\max }\right)$ corresponding to elastograms of the normal vessel in Fig. 4 (1st and 2nd rows). For each radial distance of $2 \mathrm{~mm}$, $3 \mathrm{~mm}$ and $5 \mathrm{~mm}$, those boxplots were obtained with a dataset of 360 strain values evenly distributed over $360^{\circ}$. Median strains were inversely related to the squared distance, in accordance to theory [10].

incompressibility elasticity assumption. We evaluated the two methods (constrained and unconstrained LSME) and compared the accuracy of different beamforming strategies, namely conventional focus imaging (Focus) and compound plane wave imaging (CPW). Overall, the constrained estimator applied on compound plane wave data (i.e., $\mathrm{CPW}+\mathrm{Inc}$ ) outperformed the other methods. Time-ensemble estimations in ultrafast CPW mode further improved performance over a wide range of applied strains. In vivo CPW imaging showed better reproducibility than standard focusing over two successive trials.

\section{A. Impacts of the Beamforming Strategy}

In non-invasive ultrasound imaging, array beamforming controls the image spatial resolution defined axially by the emitted pulse length, and laterally by the F-number:

$$
F_{\#}=\frac{z_{f}}{a}
$$
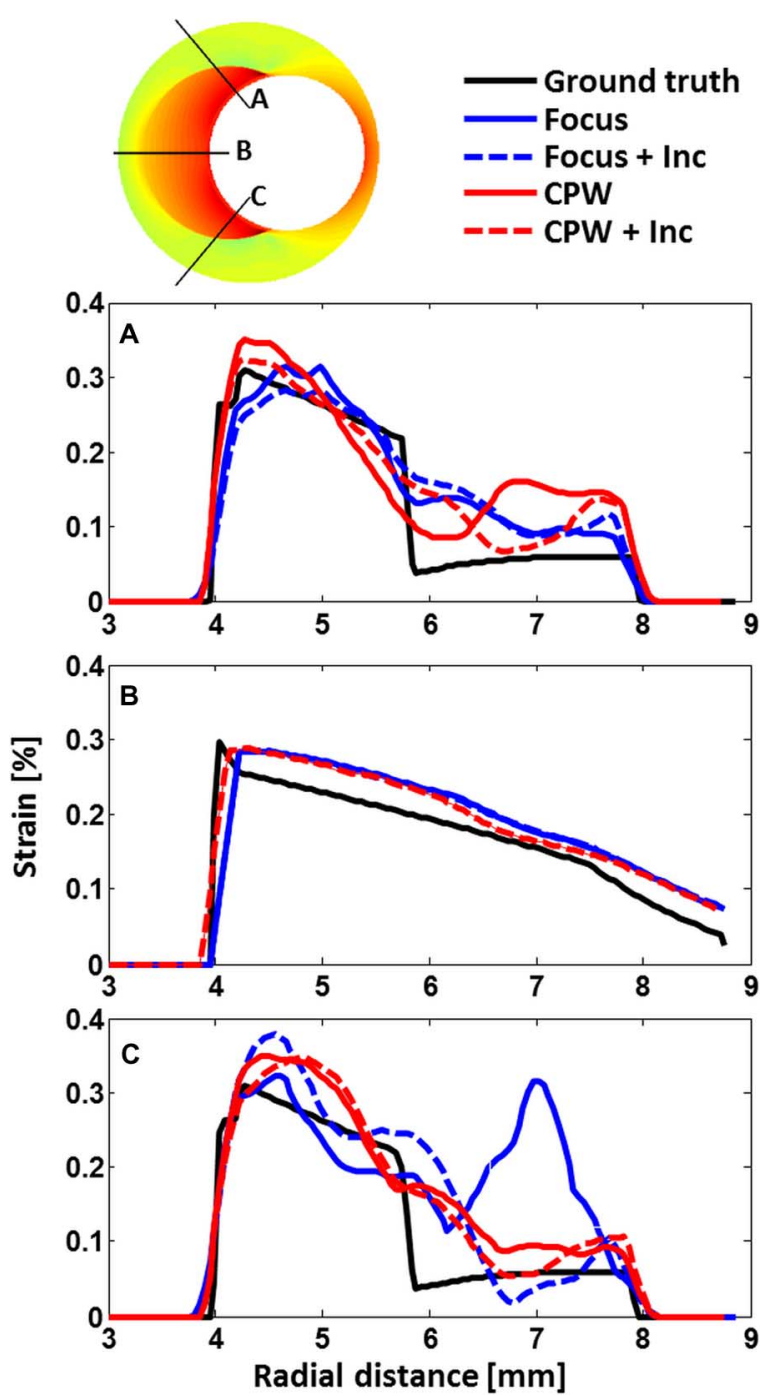

Fig. 6. Maximum principal strain $\left(\varepsilon_{\max }\right)$ profiles of the plaque model computed with the finite element model (Ground truth), and with four beamforming strategies (Focus, Focus+Inc, CPW, CPW+Inc). Profiles (A, B, C) were selected to show the robustness of the method at the edge of the simulated lipid pool.

where $z_{f}$ is the focalization depth and $a$ the size of the aperture. The smaller the F-number, the better is the lateral resolution. Dynamic focalization (i.e., changing the aperture size and focalization as a function of depth, for a constant F-number) theoretically provides the best image resolution. This can be achieved in both transmit and receipt. However, with a conventional imaging sequence (i.e., focus imaging), dynamic focalization in transmit, using for example 10 focus depths, requires 10 successive transmissions per image line. Such process decreases the frame rate by a factor equal to the number of focuses, which is not convenient for the imaging of moving structures.

Using $N$ tilted plane wave transmissions, from $-\alpha_{\max }$ to $\alpha_{\max }$, with an angular step $\Delta \alpha=\lambda / L$ between successive transmissions ( $\lambda$ being the wavelength and $L$ the full aperture of the probe), coherent plane wave compounding approximates the dynamic focalization process in transmit (see (3) and (4) in 


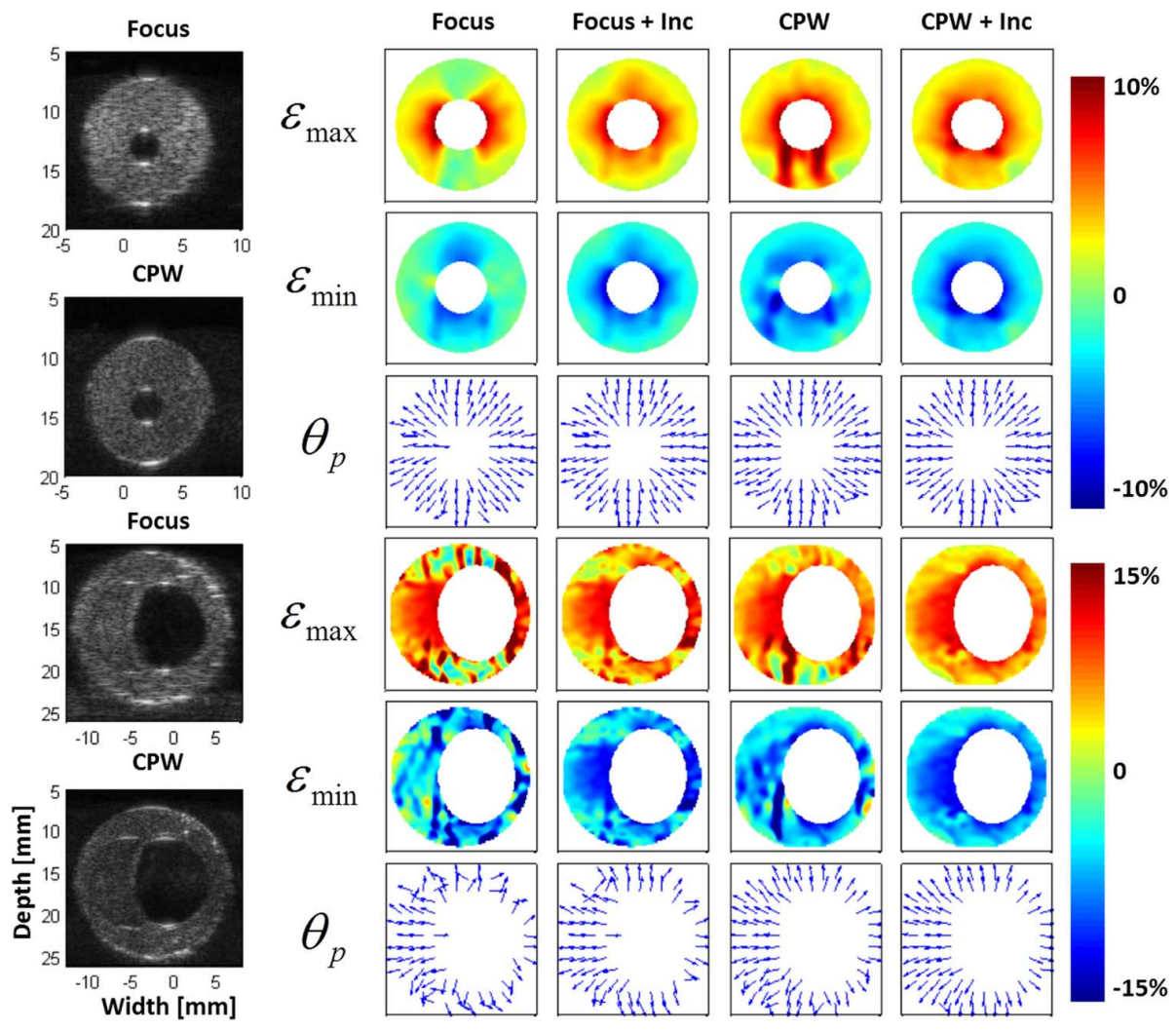

Fig. 7. B-mode images (first column), maximum, minimum and orientation of the principal strain tensor $\left(\varepsilon_{\max }, \varepsilon_{m i n}, \theta_{p}\right)$ computed on mimicking vessel phantoms with four beamforming strategies (Focus, Focus+Inc, CPW, CPW+Inc). The first three rows display results of the concentric homogeneous vessel, whereas the last three rows correspond to results of the plaque geometry.
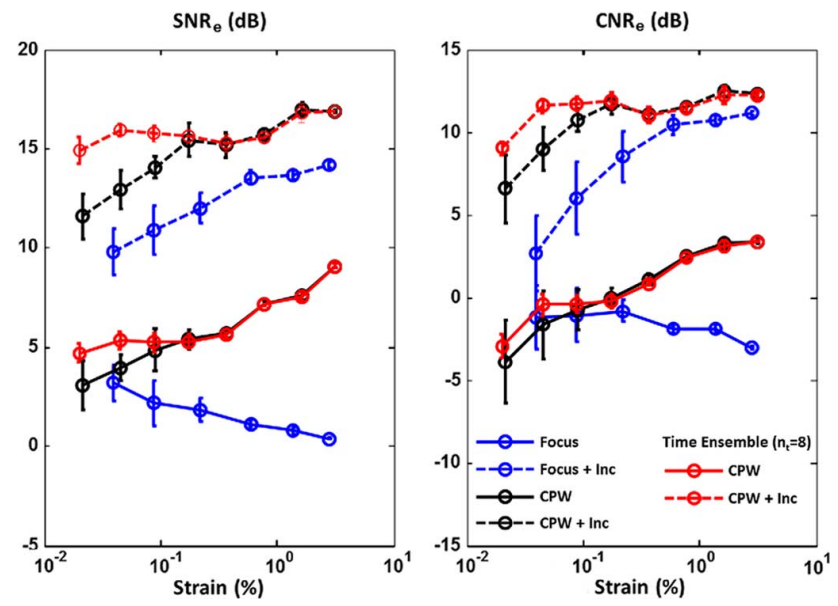

Fig. 8. Elastographic signal-to-noise ratio $\left(\mathrm{SNR}_{\mathrm{e}}\right)$ and contrast-to-noise ratio $\left(\mathrm{CNR}_{\mathrm{e}}\right)$ for $\varepsilon_{\max }$ obtained with dynamic experimental data on the homogeneous vessel mimicking phantom. $\mathrm{SNR}_{\mathrm{e}}$ and $\mathrm{CNR}_{\mathrm{e}}$ metrics, computed using Eqs. (20) and (21), are displayed as a function of the average strain, measured on the proximal circle of radius $r=2.5 \mathrm{~mm}$ for all four beamforming strategies (Focus, Focus+Inc, CPW and CPW+Inc). The impact of the time-ensemble averaging approach for $\mathrm{CPW}$ is displayed in red.

[17]). Using such approximation, the F-number in transmission becomes (see (6) in [17]):

$$
\mathrm{F}_{\#} \approx \frac{1}{\mathrm{~N} \Delta \alpha}=\frac{\mathrm{N}-1}{\mathrm{~N}} \frac{1}{2 \alpha_{\max }} \approx \frac{1}{2 \alpha_{\max }} .
$$

Note that for a single non-steered plane wave emission, the F-number in transmission would be infinite, meaning that no focalization would be performed.

In the current study, to achieve an F-number of 1.75 with the L14-5/38 probe, $N \approx 100$ steered plane waves with a maximum steering angle $\left|\alpha_{\max }\right|=16^{\circ}$ (i.e., $\Delta \alpha \approx 0.3^{\circ}$ ) would be needed. To limit the number of plane wave transmissions (i.e., to keep a high frame rate) while preserving the lateral resolution (i.e., small F-number), one can increase the angular step $\Delta \alpha$ while keeping the same $\alpha_{\max }$ (see (22)). Increasing the angular step, however, creates more adverse grating lobes in transmit (see (7) and Fig. 1 in [17]) that might affect strain estimations. We indeed found that $\alpha_{\max }$ and $\Delta \alpha$ had an impact on the quality of elastograms (Fig. 3). Increasing $\left|\alpha_{\max }\right|$ up to $10^{\circ}$ to improve the lateral resolution also provided the best quality of RF data and elastograms. Although changing the angular step $\Delta \alpha$ for $\left|\alpha_{\max }\right|=10^{\circ}$ barely influenced $\mathrm{SNR}_{\mathrm{e}}$ and $\mathrm{CNR}_{\mathrm{e}}$, it had an impact on noise levels and grating lobe interferences of beamformed RF images (Fig. 3). This can be explained by the reduced number of transmits for compounding. Interestingly, these deleterious contributions had less influence on elastograms likely because of the robustness of the LSME estimator to noise.

Note that grating lobes had more impact on CPW beamformed RF images than noise (SIR $\sim 25 \mathrm{~dB}, \mathrm{SNR} \sim 50 \mathrm{~dB}$ ). Grating lobes are the main source of artifacts in strain imaging and should be minimized, either by increasing the number of plane wave transmissions (small $\Delta \alpha$ ), at the expense of the frame rate, or by using larger F-numbers (small $\alpha_{\max }$ ), but at the expense of the lateral resolution. 


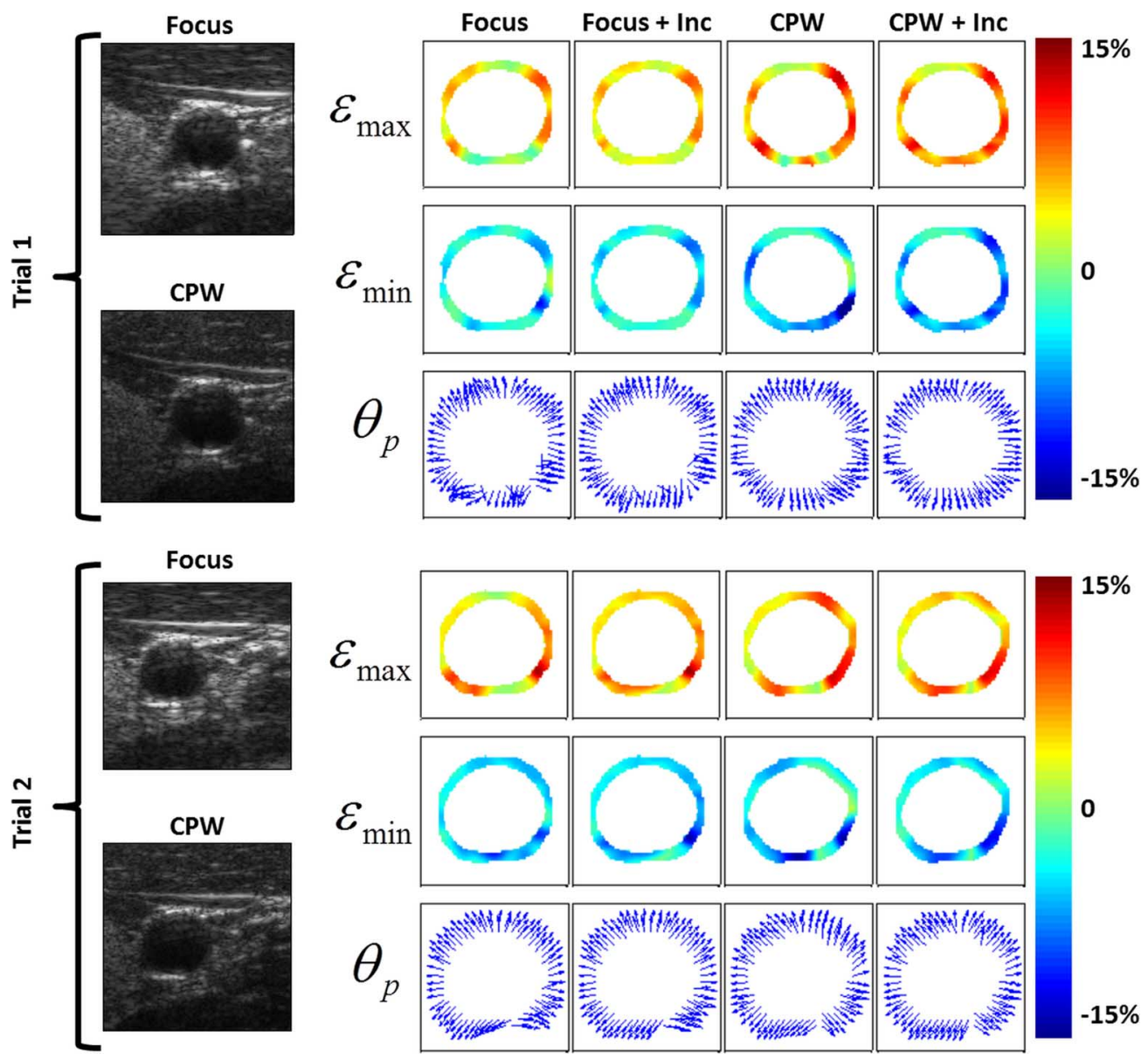

Fig. 9. B-mode images (first column) of the common carotid artery of a healthy volunteer in a transverse plane using both Focus and CPW imaging. Principal components $\left(\varepsilon_{\max }, \varepsilon_{\min }\right)$ and orientation $\left(\theta_{p}\right)$ of the strain tensor for all four beamforming strategies (Focus, Focus+Inc, CPW, CPW+Inc) are displayed at the time of maximum cumulated deformations (see Fig. 10). Results for CPW imaging were computed with the time-ensemble averaging method using $n_{t}=8$ successive pairs of frames.

To deal with the lack of lateral resolution of ultrasound systems, pioneering strain compounding strategies using either focus [9], [10] or plane wave beam steering [11] were proposed. However, as they require large beam-steered angles (at least $15^{\circ}$ ), those methods were not explored in this study. In fact, beam-steering at large angles with linear array transducers creates strong adverse grating lobe interferences that need to be low pass filtered, as described in [10]. The following discussion aims to explain why this would have not been possible in the current study.

The angular position of grating lobes (from the main lobe) is defined by [46]:

$$
\theta_{g}=\sin ^{-1}\left(\sin (\alpha) \pm \frac{\lambda}{D}\right)
$$

where $\alpha$ is the beam steering angle and $D$ the pitch of the probe. In the case of the L14-5/38 probe (Ultrasonix Medical Corp.), a steering at $|\alpha|=15^{\circ}$ with $D=0.3048 \mathrm{~mm}$ would produce grating lobes at $\theta_{g}=26^{\circ}$. The following equation explains the cut-off frequency of the low-pass filter that would be required; it can be deduced from (24):

$$
f_{\text {cut }-o f f}=\frac{c}{(1+\sin (\alpha)) \cdot D}
$$
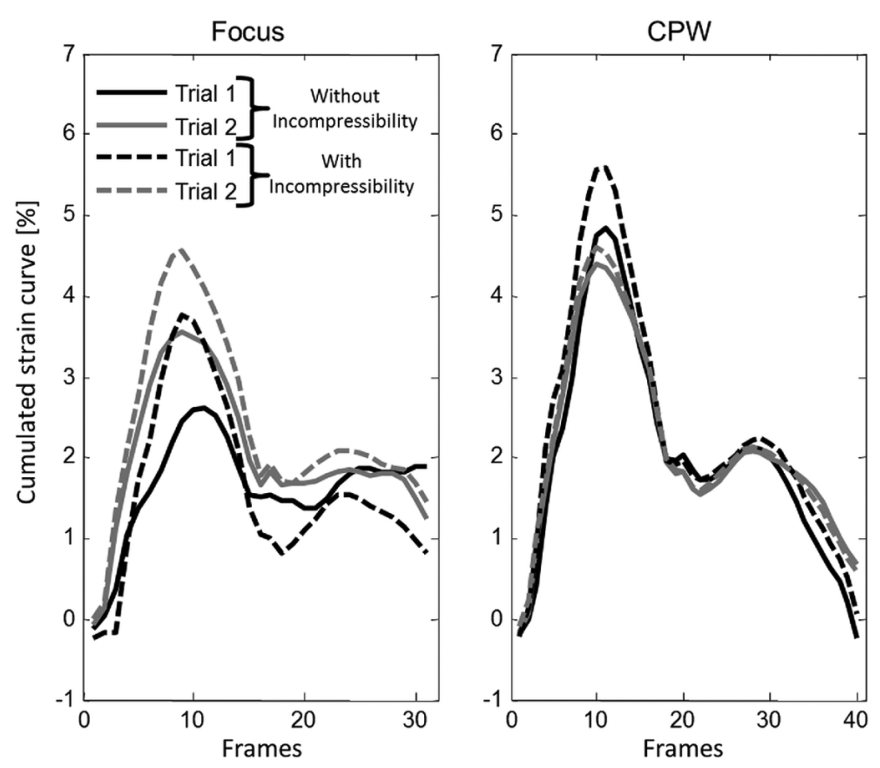

Fig. 10. Spatially averaged cumulated maximum principal strain curves for both focus and compound plane wave imaging methods, with and without the plane strain incompressibility constrained estimator over one cardiac cycle for two successive trials. For CPW data, the time-ensemble approach was used $\left(n_{t}=8\right)$ to improve the robustness of the algorithm. 
where $c$ is the speed of sound. For $|\alpha|=15^{\circ}$ this would correspond to $f_{\text {cut-off }} \approx 4 \mathrm{MHz}$. As the bandwidth of the transducer is between $4-10 \mathrm{MHz}$ (7.2 MHz central frequency), the low pass filter would have removed most of the information and this approach was thus ignored in our study.

The proposed CPW method would benefit from additional strain compounding [11] (i.e., averaging strain images estimated at different steered angle). However, such technique requires a relatively small pitch (ideally $\lambda / 2$ ) to avoid adverse effects of grating lobes inside the field of view.

\section{B. Advantages of the Plane-Strain Incompressibility Constraint}

The use of the incompressibility kinematics constraint has already been proposed in the context of quasi-static elastography to overcome the effect of the low lateral resolution in ultrasound imaging [9], [10]. In those studies, the incompressibility assumption was used to perform angular strain compounding from beam steered data. The novelty of our approach lies in the formulation of the problem to include this hypothesis with the plane strain assumption into the motion model. This new formulation of the Lagrangian speckle model estimator (LSME) reduces the complexity of the algorithm: the number of unknowns drops from nine to six with the plane strain assumption, and further from six to five when assuming incompressibility (see II-C-2). This makes the LSME more robust to speckle incoherences associated with the low lateral resolution. In this study, the use of the constrained estimator decreased the variability and thus increased the contrast between soft and hard tissue components (Figs. 4 and 7).

Plane strain incompressibility is a widely accepted hypothesis in biomechanics when recovering the apparent Young modulus map from 2D strains [29], [30], since out-of-plane components cannot be measured with 2D estimators. As discussed in II-C-2, this assumption stands when carotid plaques and inclusions are wider (along the vessel axis) than thicker (radially) [32]. For more complex vascular geometries, such assumptions might be violated. In those situations, precision improvement (i.e., less variance) with the proposed constrained estimator would be at the expense of accuracy (i.e., larger bias). However, in the perspective of clinical applications where we intend to discriminate hard and soft plaque components, the proposed method would still be of interest as it significantly improved contrastand signal-to-noise ratios. Using 3D acquisition probes as in [47], the ambiguity associated with the projection of an actual 3D tensor would be solved using the incompressibility assumption without plane strain condition.

\section{Impact of the Frame Rate on Non-Invasive Vascular Elastography}

One of the main issue in strain imaging is the inability of conventional strain estimators to perform accurate estimations of very small $(<0.1 \%)$ or very high $(>10 \%)$ deformations [13]. The strain filter, which describes the elastographic signal-tonoise ratio $\left(\mathrm{SNR}_{\mathrm{e}}\right)$ as a function of applied strains, defines the range of deformations that can be accurately estimated for a given estimator. Using optical flow methods, the variance decreases when the number of constraints ((1)) increases. When using a single pair of RF images, the number of constraints can be increased by extending the spatial support (MW) of the estimator, but at the expense of the spatial resolution of strain maps. When using high frame rate imaging systems (here we obtained effective frame rates of $\sim 500 \mathrm{~s}^{-1}$ with the CPW beamforming strategy versus $\sim 50 \mathrm{~s}^{-1}$ for the Focus sequence), the number of constraints can be increased by extending the support through time (i.e., $\mathrm{MW}_{\mathrm{t}}$ ). Using this time-ensemble estimation approach, we showed (Fig. 8) that CPW imaging could reach better $\mathrm{SNR}_{\mathrm{e}}$ and $\mathrm{CNR}_{\mathrm{e}}$, especially when estimating small deformations. This can be of importance when evaluating biological tissues under small stress conditions and/or hard components (e.g., a fibrous carotid plaque) with fewer deformations than surrounding softer tissues. Furthermore, it is important to recall that optical flow methods are subjected to aliasing artifacts [22]. The use of ultrafast imaging can prevent such artifacts by keeping the inter-frame time step $\Delta t$ and thus the inter-frame motion small enough.

\section{In Vivo Feasibility}

We found good reproducibility in vivo when using CPW imaging (Figs. 9 and 10). The combination of ultrafast compound plane wave imaging with the constrained LSME estimator provided reliable plane strain tensors in contrast to standard focusing giving strain orientations inconsistent with the radial blood pressure direction. Still, strain maps appeared inhomogeneous circumferentially whatever the beamforming method. Such behaviour, in the case of a healthy artery, could be explained by differences in boundary conditions. In fact, the far wall of the artery is surrounded by more rigid structures (paraspinal muscles and cervical spine) than the near wall, mainly surrounded by the sternocleidomastoid muscle and compliant jugular vein in an antero lateral position. For simulations and in vitro experiments (Figs. 4 and 7, respectively), boundary conditions were uniform circumferentially. Further study on the impact of boundary conditions would be of interest for a better understanding of the vascular wall strain behaviour in vivo.

Vascular strain analysis has recently gained a lot of interest [48]-[54] as elevated shear movements might be associated with plaque development leading to its rupture. For examples, recent in vivo studies using endovascular elastography (EVE) on a diabetic pig model [51] and on humans, prior and following coronary atherectomy interventions [52] revealed the potential of radial shear strains to detect and classify atherosclerotic plaques according to American Heart Association classes [55]. Those studies also revealed a significant correlation between the radial shear strain magnitude and the level of inflammation and soft plaque content. With the proposed method ( $\mathrm{CPW}+$ Inc+time-ensemble averaging), we are now able to estimate all components of the plane strain tensor. With our previous implementation [7], we were only able to reliably estimate the axial strain and shear in the case of NIVE, and radial strain and shear in the case of EVE [52]. The current LSME implementation may be extended to EVE (using standard intravascular ultrasound focusing scheme) and help understanding the complex mechanical behavior of coronary arteries. It may also be possible to implement the 
proposed LSME method for multi-physics imaging of coronary atherosclerotic plaques [56].

\section{E. Study Limitations}

A few limitations deserve to be pointed out, even if the present study does highlight original and potentially promising concepts for improving non-invasive atherosclerotic plaque identification from ultrasound strain measurements.

- Because 2D ultrasound images were acquired, our structural analysis was performed in two dimensions assuming negligible out-of-plane motion. Note that compound plane wave imaging, proposed here in the context of 2D NIVE, can be extended to 3D acquisitions using 2D matrix arrays [57]. Our method would certainly benefit from this innovative technique, as it would allow the estimation of the full 3D strain tensor.

- The plane strain hypothesis, here combined with incompressibility to mitigate the impact of the low lateral resolution, is a strong assumption when dealing with arteries with typically $70 \%$ stenosis or greater. Even if such assumption is mechanically reasonable insofar as long as the plaque length is large with regard to the radial dimension of the artery [5], [28], [29], [58], it probably does not hold for complex geometries as discussed earlier (V-B). In the near future, using $3 \mathrm{D}$ acquisition systems, the incompressibility constraint could be used without the plane strain assumption and thus be applied to complex geometries.

- Although the incompressibility assumption introduced in our model allowed dealing with the inability of non-invasive ultrasound to estimate lateral strains accurately, no mechanical assumption was introduced to further improve lateral shear components. Improvements of the methodology, using beam steering [9], [10] methods, may further improve strain and shear estimations. However, as stated earlier (V-A), linear array transducers with small pitch $(\lambda / 2)$ should be used instead of conventional linear arrays.

- The spatial resolution of estimated principal strain maps is limited by the spatial resolution of the transducer (which is on the order of half a wavelength axially, i.e. $\lambda / 2 \approx 100 \mu \mathrm{m}$ and $\approx 400 \mu \mathrm{m}$ laterally), and by the spatial support of the estimator (FWHM $=1 \mathrm{~mm}$ in the current study). Structures smaller than $1 \mathrm{~mm}$ may not be completely discriminated using the proposed method. However, non-invasive elastography using high frequency probes is feasible and has been demonstrated in vitro and in vivo [59]. Further developments in high frequency ultrasound imaging using array transducers may provide plane wave insonification and higher resolution elastograms.

\section{CONCLUSION}

Ultrafast compound plane wave imaging combined with a model-based elastography technique provided accurate strain elastograms of mimicked arteries (in silico and in vitro), and a proof-of-concept in an in vivo case was presented. We found that the proposed constrained estimator method $(\mathrm{CPW}+\mathrm{Inc})$ outperformed conventional focus and CPW methods. Compound plane wave imaging could provide accurate principal strain components with a frame rate 10 to 20 times higher than conventional NIVE methods. The increased frame rate allowed further improvements using time-ensemble estimation in the proposed LSME framework. We also demonstrated that the CPW+Inc method could estimate $0.02 \%$ to $3.2 \%$ strains accurately, which may be of value when evaluating diseased arteries with heterogeneous rigidity components.

\section{ACKNOWLEDGMENT}

The authors would like to thank Dr. F. Destrempes for providing mathematical proofs to reviewers.

\section{REFERENCES}

[1] W. Casscells, M. Naghavi, and J. T. Willerson, "Vulnerable atherosclerotic plaque," Circulat. Res., vol. 107, pp. 2072-2075, 2003.

[2] T. J. Kaufmann and D. F. Kallmes, "Utility of MRA and CTA in the evaluation of carotid occlusive disease," Seminars Vasc. Surg., pp. 75-82, 2005.

[3] A. Fenster, C. Blake, I. Gyacskov, A. Landry, and J. D. Spence, "3D ultrasound analysis of carotid plaque volume and surface morphology," Ultrasonics, vol. 44, pp. 153-157, 2006.

[4] C. Naim et al., "Vulnerable atherosclerotic carotid plaque evaluation by ultrasound, computed tomography angiography, magnetic resonance imaging: An overview," Can. Assoc. Radiol. J., vol. 65, pp. 275-286, 2014.

[5] G. Finet, J. Ohayon, and G. Rioufol, "Biomechanical interaction between cap thickness, lipid core composition and blood pressure in vulnerable coronary plaque: Impact on stability or instability," Coronary Artery Disease, vol. 15, pp. 13-20, 2004.

[6] M. Naghavi, Asymptomatic Atherosclerosis: Pathophysiology, Detection and Treatment. New York: Springer Science Business Media, 2010.

[7] E. Mercure et al., "A local angle compensation method based on kinematics constraints for non-invasive vascular axial strain computations on human carotid arteries," Comput. Med. Imag. Graph., vol. 38, pp. 123-136, 2014.

[8] E. Konofagou and J. Ophir, “A new elastographic method for estimation and imaging of lateral displacements, lateral strains, corrected axial strains and Poisson's ratios in tissues," Ultrasound Med. Biol., vol. 24, pp. 1183-1199, 1998.

[9] M. Rao et al., "Normal and shear strain estimation using beam steering on linear-array transducers," Ultrasound Med. Biol., vol. 33, pp. 57-66, 2007.

[10] H. Hansen, R. Lopata, and C. L. de Korte, "Noninvasive carotid strain imaging using angular compounding at large beam steered angles: Validation in vessel phantoms," IEEE Trans. Med. Imag., vol. 28, no. 6, pp. 872-880, Jun. 2009

[11] H. H. G. Hansen, A. E. C. M. Saris, N. R. Vaka, M. M. Nillesen, and C. L. de Korte, "Ultrafast vascular strain compounding using plane wave transmission," J. Biomechan., vol. 47, pp. 815-823, 2014.

[12] J. Vappou, J. Luo, and E. E. Konofagou, "Pulse wave imaging for noninvasive and quantitative measurement of arterial stiffness in vivo," Am. J. Hypertension, vol. 23, pp. 393-398, 2010.

[13] T. Varghese, J. Ophir, E. Konofagou, F. Kallel, and R. Righetti, "Tradeoffs in elastographic imaging," Ultrason. Imag., vol. 23, pp. 216-248, 2001.

[14] G. Montaldo, M. Tanter, J. Bercoff, N. Benech, and M. Fink, "Coherent plane-wave compounding for very high frame rate ultrasonography and transient elastography," IEEE Trans. Ultrason., Ferroelectr. Freq. Control, vol. 56, no. 3, pp. 489-506, Mar. 2009.

[15] J. A. Jensen, S. I. Nikolov, K. L. Gammelmark, and M. H. Pedersen, "Synthetic aperture ultrasound imaging," Ultrasonics, vol. 44, pp. $5-15,2006$.

[16] S. Korukonda et al., "Noninvasive vascular elastography using planewave and sparse-array imaging," IEEE Trans. Ultrason., Ferroelectr. Freq. Control, vol. 60, no. 2, pp. 332-342, Feb. 2013.

[17] B. Denarie et al., "Coherent plane wave compounding for very high frame rate ultrasonography of rapidly moving targets," IEEE Trans. Med. Imag., vol. 32, no. 7, pp. 1265-1276, Jul. 2013.

[18] M. A. Lubinski, S. Y. Emelianov, and M. O'Donnell, "Speckle tracking methods for ultrasonic elasticity imaging using short-time correlation," IEEE Trans. Ultrason., Ferroelectr. Freq. Control, vol. 46, no. 1, pp. 82-96, Jan. 1999. 
[19] R. L. Maurice et al., "Noninvasive vascular elastography: Theoretical framework," IEEE Trans. Med. Imag., vol. 23, no. 2, pp. 164-180, Feb. 2004.

[20] H. Liebgott, A. Basarab, P. Gueth, D. Friboulet, and P. Delachartre, "Transverse oscillations for tissue motion estimation," Ultrasonics, vol. 50, pp. 548-555, 2010.

[21] C. Schmitt, G. Soulez, R. L. Maurice, M.-F. Giroux, and G. Cloutier, "Noninvasive vascular elastography: Toward a complementary characterization tool of atherosclerosis in carotid arteries," Ultrasound Med. Biol., vol. 33, pp. 1841-1858, 2007.

[22] D. Fleet and Y. Weiss, "Optical flow estimation," in Handbook of Mathematical Models in Computer Vision. New York: Springer, 2006, pp. 237-257.

[23] R. L. Maurice and M. Bertrand, "Speckle-motion artifact under tissue shearing," IEEE Trans. Ultrason., Ferroelectr. Freq. Control, vol. 46, no. 3, pp. 584-594, May 1999.

[24] E. Mercure, G. Cloutier, C. Schmitt, and R. L. Maurice, "Performance evaluation of different implementations of the Lagrangian speckle model estimator for non-invasive vascular ultrasound elastography," Med. Phys., vol. 35, pp. 3116-3126, 2008.

[25] P. W. Holland and R. E. Welsch, "Robust regression using iteratively reweighted least-squares," Commun. Stat. Theory Methods, vol. 6, pp. 813-827, 1977.

[26] T. E. Carew, R. N. Vaishnav, and D. J. Patel, "Compressibility of the arterial wall," Circulat. Res., vol. 23, pp. 61-68, 1968.

[27] G. A. Holzapfel, "Structural and numerical models for the (visco) elastic response of arterial walls with residual stresses," Biomechan. Soft Tissue Cardiovasc. Syst., vol. 441, pp. 109-184, 2003.

[28] R. T. Lee et al., "Computational structural analysis based on intravascular ultrasound imaging before in vitro angioplasty: Prediction of plaque fracture locations," J. Am. Coll. Cardiol., vol. 21, pp. 777-782, 1993.

[29] S. Le Floc'h et al., "On the potential of a new IVUS elasticity modulus imaging approach for detecting vulnerable atherosclerotic coronary plaques: In vitro vessel phantom study," Phys. Med. Biol., vol. 55, pp. 5701-5721, 2010.

[30] R. A. Baldewsing, J. A. Schaar, F. Mastik, C. W. Oomens, and A. F. van der Steen, "Assessment of vulnerable plaque composition by matching the deformation of a parametric plaque model to measured plaque deformation," IEEE Trans. Med. Imag., vol. 24, no. 4, pp. 514-528, Apr. 2005.

[31] H. Ribbers et al., "Noninvasive two-dimensional strain imaging of arteries: Validation in phantoms and preliminary experience in carotid arteries in vivo," Ultrasound Med. Biol., vol. 33, pp. 530-540, 2007.

[32] J. Ohayon, G. Finet, F. Treyve, G. Rioufol, and O. Dubreuil, "A three dimensional finite element analysis of stress distribution in a coronary atherosclerotic plaque: In vivo prediction of plaque rupture location," Biomechan. Appl. Comput. Assist. Surg., vol. 37, pp. 225-241, 2005.

[33] K. K. Shung, Diagnostic Ultrasound: Imaging and Blood Flow Measurements. Boca Raton, FL: CRC press, 2005.

[34] C. D. Meinhart, S. T. Wereley, and J. G. Santiago, "A PIV algorithm for estimating time-averaged velocity fields," J. Fluids Eng., vol. 122, pp. 285-289, 2000.

[35] S. A. V. D. Geer, "Least squares estimation," in Encyclopedia of Statistics in Behavioral Science. New York: Wiley, 2005, vol. 2, pp. 1041-1045

[36] F. Destrempes, M.-H. Roy Cardinal, L. Allard, J.-C. Tardif, and G. Cloutier, "Segmentation method of intravascular ultrasound images of human coronary arteries," Comput. Med. Imag. Graph., vol. 38, pp. 91-103, 2014.

[37] D. Garcia, "A fast all-in-one method for automated post-processing of PIV data," Exper. Fluids, vol. 50, pp. 1247-1259, 2011.

[38] D. Beattie, C. Xu, R. Vito, S. Glagov, and M. C. Whang, "Mechanical analysis of heterogeneous, atherosclerotic human aorta," J. Biomechan. Eng., vol. 120, pp. 602-607, 1998.
[39] R. F. Wagner, S. W. Smith, J. M. Sandrik, and H. Lopez, "Statistics of speckle in ultrasound B-scans," IEEE Trans. Sonics Ultrason., vol. 30, no. 3, pp. 156-163, May 1983.

[40] J. A. Jensen, "Field: A program for simulating ultrasound systems," in Proc. 10th Nordicbaltic Conf. Biomed. Imag., 1996, pp. 351-353.

[41] J. Fromageau, J.-L. Gennisson, C. Schmitt, R. L. Maurice, R. Mongrain, and G. Cloutier, "Estimation of polyvinyl alcohol cryogel mechanical properties with four ultrasound elastography methods and comparison with gold standard testings," IEEE Trans. Ultrason., Ferroelectr. Freq. Control, vol. 54, no. 3, pp. 498-509, Mar. 2007.

[42] G. T. Mase and G. E. Mase, Continuum Mechanics for Engineers. London, U.K.: CRC Press, 1992.

[43] D. Garcia et al., "Stolt's f-k migration for plane wave ultrasound imaging," IEEE Trans. Ultrasonics, Ferroelectrics and Frequency Control, vol. 60, no. 9, pp. 1853-1867, Sep. 2013.

[44] J. Allen, "Photoplethysmography and its application in clinical physiological measurement," Physiol. Meas., vol. 28, pp. R1-R39, 2007.

[45] C. Naim et al., "Characterisation of carotid plaques with ultrasound elastography: Feasibility and correlation with high-resolution magnetic resonance imaging," Eur. Radiol., vol. 23, pp. 2030-2041, 2013.

[46] J. Huang, P. W. Que, and J. H. Jin, "A parametric study of beam steering for ultrasonic linear phased array transducer," Russ. J. Nondestruct. Test., vol. 40, pp. 254-259, 2004.

[47] G. M. Treece, J. E. Lindop, A. H. Gee, and R. W. Prager, "Freehand ultrasound elastography with a 3-D probe," Ultrasound Med. Biol., vol. 34, pp. 463-474, 2008.

[48] T. Idzenga, G. Pasterkamp, and C. L. de Korte, "Shear strain in the adventitial layer of the arterial wall facilitates development of vulnerable plaques," Bioscience Hypotheses, vol. 2, pp. 339-342, 2009.

[49] M. Cinthio et al., "Longitudinal movements and resulting shear strain of the arterial wall," Am. J. Physiol.-Heart Circulat. Physiol., vol. 291, pp. 394-402, 2006.

[50] T. Idzenga, S. Holewijn, H. H. G. Hansen, and C. L. de Korte, "Estimating cyclic shear strain in the common carotid artery using radiofrequency ultrasound," Ultrasound Med. Biol., vol. 38, pp. 2229-2237, 2012.

[51] Y. Majdouline et al., "Endovascular shear strain elastography for the detection and characterization of the severity of atherosclerotic plaques: In vitro validation and in vivo evaluation," Ultrasound Med. Biol., vol. 40, pp. 890-903, 2014.

[52] Z. Keshavarz-Motamed et al., "Coronary artery atherectomy reduces plaque shear strains: An endovascular elastography imaging study," Atherosclerosis, vol. 235, pp. 140-149, 2014.

[53] M. Larsson et al., "Strain assessment in the carotid artery wall using ultrasound speckle tracking: Validation in a sheep model," Phys. Med. Biol., vol. 60, p. 1107, 2015.

[54] R. G. P. Lopata et al., "Performance evaluation of methods for twodimensional displacement and strain estimation using ultrasound radio frequency data," Ultrasound Med. Biol., vol. 35, pp. 796-812, 2009.

[55] H. C. Stary, "Natural history and histological classification of atherosclerotic lesions an update," Arteriosclerosis, Thrombosis, Vasc. Biol., vol. 20, pp. 1177-1178, 2000.

[56] M. Abran et al., "Development of a photoacoustic, ultrasound and fluorescence imaging catheter for the study of atherosclerotic plaque," IEEE Trans. Biomed. Circuits Syst., vol. 8, no. 5, pp. 696-703, Oct. 2014.

[57] J. Provost et al., "3D ultrafast ultrasound imaging in vivo," Phys. Med. Biol., vol. 59, pp. L1-L13, 2014.

[58] H. M. Loree, R. D. Kamm, R. G. Stringfellow, and R. T. Lee, "Effects of fibrous cap thickness on peak circumferential stress in model atherosclerotic vessels," Circulation Res., vol. 71, pp. 850-858, 1992.

[59] R. L. Maurice et al., "Non-invasive high-frequency vascular ultrasound elastography," Phys. Med. Biol., vol. 50, pp. 1611-1628, 2005. 